\title{
Diagnostics and modeling of plasma processes in ion sources
}

\author{
Akos Vertes*, Renaat Gijbels, and Fred Adams \\ Department of Chemistry, University of Antwerp (U.I.A.), Universiteitsplein 1, \\ B-2610 Wilrijk, Belgium
}

\section{INTRODUCTION}

Plasma ion sources are constantly growing in importance in the field of atomic mass spectrometry. The idea is to deposit enough energy into the analyte to atomize and ionize a representative part of it and to achieve a plasma state that will endure for some period of time. A fraction of the ions can then be extracted from the plasma and analyzed in the mass spectrometer. The main difference among the methods for plasma ionization lies in the form in which energy is delivered to the sample. Either it is in the form of electric current (glow discharge, spark) or it is mediated by electromagnetic radiation (inductively coupled plasma (ICP), microwave-induced plasma (MIP), pulsed laser). The present state of the art in using these sources in analytical applications is well summarized in a recent review (1) and in a recently published monograph (2).

Plasma ion sources are not only used in mass spectrometers, but have become essential parts of ion implanters, accelerators, nuclear fusion devices, etc. (3). For ion implantation, ion milling, and etching purposes, hollow cathode (4), plasma cathode (5-8), radiofrequency (9) and microwave ion sources (10-12) are under intense investigation. Diligent research is devoted to the ever-improving ion sources for accelerator and nuclear fusion applications. Multicusp (13-16), surface plasma (17), hollow cathode (18), microwave (19), alkaline anode (20-22), radial field discharge (23), and micropinch (24) sources are among the subjects of current interest. We refer to the multitude of these plasma ion source construction efforts only because-as has happened already many times before-ne:v mass spectrometric sources may emerge from such developments. Of these methods hollow cathode sources are familiar to the analytical spectroscopist, and microwave sources are just about to debut in mass spectrometry.

The expected properties of the source depend on the particular demands of

${ }^{*}$ To whom correspondence should be addressed. Phone: [323] 820.23.82. Electronic mail: VERTES@BANUIA52.BITNET. On leave from: Central Research Institute for Physics of the Hungarian Academy of Sciences.

Mass Spectrometry Reviews 1990, 9, 71-113

(C) 1990 John Wiley \& Sons, Inc. 
the application. In ion implanters, high currents of a certain ionic species with homogeneous beam cross section are necessary, whereas in analytical mass spectrometry, representative beams are favorable (i.e., those in which the ionic composition corresponds as closely as possible to that of the sample). High beam intensity and the lack of electrode corrosion are general requirements in many applications. In accord with the mission of the journal, we restrict our discussion to ion sources common in mass spectrometry.

The most essential step in every ionization process is the conversion of neutral particles into ions, it is therefore virtually unavoidable to pass through a phase where neutrals and ions coexist. This phase can be described by the very general term: plasma. There are indications for the existence of a transient and/or local plasma in such techniques as secondary ionization (SIMS), fast atom bombardment (FAB), and ${ }^{252} \mathrm{Cf}$ plasma desorption mass spectrometry. For practical reasons, however, we will confine the discussion to ionization methods where the plasma itself actively takes part in the ionization process (i.e., those in which ionneutral collisions cannot be neglected).

To satisfy requirements for design and efficient operation of plasma ion sources, researchers have initiated efforts to understand and describe the underlying physicochemical processes. Plasma diagnostic methods were borrowed from plasma physics to measure temperature and number density distributions of different specics inside particular ion sources. $\Lambda$ comprehensive survey of these spectroscopic, microwave, laser and electrical methods can be found in Ref. 25. Further possibilities are derived from the mathematical solution of plasma models.

Section II is an outline of the methods of plasma diagnostics as they were tailored to cope with the special problems in mass spectrometric ion sources. We discuss among them: (A) electrical probes, (B) optical methods, (C) ion extraction and analysis, (D) mathematical modeling, and (E) miscellaneous modes of attaining information on the plasma state. Almost all plasma ion sources were used previously as optical emission sources (spark, glow discharge, ICP). Considerable knowledge, therefore, can be transferred from investigations of the corresponding optical sources and can be compiled together with results obtained on the mass spectrometric sources.

In Section III, we survey and report efforts in diagnostics and modeling of several plasma sources as used in mass spectrometry. Generally speaking, two main types of sources can be separated on the basis of their behavior in time. Stationary sources reach time-independent density and temperature distributions after a short transient period, whereas transient sources do not reach a stationary state. In Section III.A, stationary regime ionization, three important methods are covered: (i) inductively coupled plasma mass spectrometry, (ii) microwave-induced plasma ion source, and (iii) glow discharge mass spectrometry. Section III deals with ionization in transient mode describing (i) spark ion source and (ii) laser plasma ionization.

\section{METHODS OF PLASMA DIAGNOSTICS AND MODELING}

To characterize a plasma we need a broad range of information:

1. The variety and concentration of all species and the rate of their production and consumption 
Table I. Working conditions of plasma ion sources

\begin{tabular}{llll}
\hline Source & \multicolumn{1}{c}{ Gas } & Pressure (Torr) & \multicolumn{1}{c}{ Excitation } \\
\hline Glow & $\mathrm{Ar}, \mathrm{Ne}$ & $0.01-10$ & $\mathrm{DC} / 13.56 \mathrm{MHz} ; 5-50 \mathrm{~W}$ \\
ICP & $\mathrm{Ar}, \mathrm{He}, \mathrm{Ar} / \mathrm{N}_{2}$ & 760 & $5-50 \mathrm{MHz} ; 1-10 \mathrm{~kW}$ \\
MWIP & $\mathrm{Ar}, \mathrm{He}, \mathrm{N}_{2}$ & 760 & $150-2450 \mathrm{MHz} ; 50-500 \mathrm{~W}$ \\
Spark & None & $10^{-6}$ & $10-100 \mathrm{~ns} ; 10^{8} \mathrm{~W} / \mathrm{cm}^{2}$ \\
Laser & None & $10^{-6}$ & $\sim 10 \mathrm{~ns} ; 10^{8}-10^{10} \mathrm{~W} / \mathrm{cm}^{2}$ \\
\hline
\end{tabular}

2. Distribution of momentum and energy and the fluxes of their sources and sinks

3. Cross sections of all possible processes involved

Each of these quantities may show spatial as well as temporal variation.

The determination of all these quantities seems, and in fact, is hopeless even for a system as simple as pure hydrogen plasma. In many cases, it is sufficient, however, to keep track of the most reactive or some species of particular interest. Therefore, in analytical applications, the bottom line is to follow the behavior of electrons and analyte ions and atoms. If complete or local thermodynamic equilibrium (LTE) prevails, the characterization of the plasma boils down to the determination of electron densities, velocities, and temperatures. The mass action law and macroscopic charge neutrality then provide the related quantities for the other components (26). To accomplish even this vastly reduced task, a wide diversity of material parameters are needed. Data on collision cross-sections, transition probabilities in the plasma phase, chemical behavior of the solid surface, and transport properties in both phases are important $(27,28)$.

The different plasma-generating mass spectrometric sources and their working conditions are listed in Table I. Glow, ICP, and MIP sources are based on stationary electric discharges supported by electric currents. In the first case, the energy is coupled to the plasma through electrodes, whereas in the case of ICP and MIP, the coupling is electromagnetic. In glow, ICP, and MIP sources, external gases with high ionization potential are used to establish stable conditions in the plasma and to ensure ionization of all species with a lower ionization potential. The spark and laser sources are based on transient plasma generation by electric breakdown and laser heating, respectively.

It is possible to locate all plasmas of interest in mass spectrometry in a so-called plasma map (Fig. 1). Estimated electron number density, $n_{c}$, and electron temperature, $T_{e}$, ranges are shown for the different sources. It is instructive to observe how stationary and transient sources are segregated in such a graph. This is because the high temperature and density of the transient plasmas cannot be sustained for extended periods of time with the present methods.

In the following sections, we briefly survey the different tools of plasma diagnostics.

\section{A. Electrical probes}

Among the most commonly used electrical probes are the one- and two-electrode systems capable of withstanding the harsh conditions prevailing in the 


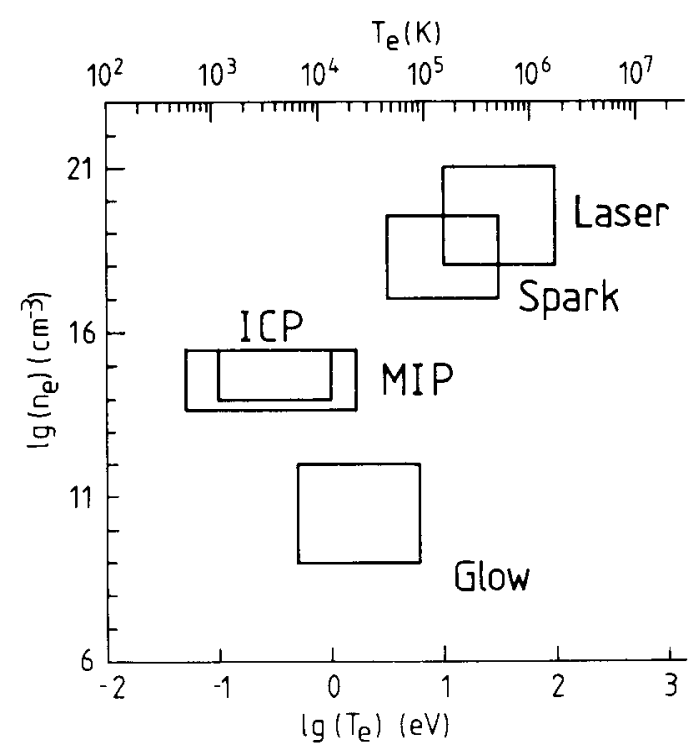

Figure 1. Approximate temperature and number density range of electrons for the different plasma sources.

plasma. The electrode(s) are connected to a driving-measuring circuit designed to determine electrode potential or current-voltage characteristics. The principle of the two-electrode setup is similar to the equipment used in electrochemistry for polarographic measurements, although serious shielding and filtering are usually necessary to diminish disturbances from the energy-delivering system sustaining the plasma. A schematic representation of this measurement for an ICP torch together with a typical current-voltage curve is shown in Figure 2.

The current through the cell, $I$, is built up of exchange currents at the electrodes:

$$
I=I_{e 1}-I_{i 1}=I_{i 2}-I_{e 2} \text {, }
$$

where $I_{e 1}, I_{i 1}, I_{e 2}$, and $I_{i 2}$ are denoting electron (e) and ion (i) current for electrodes 1 and 2 , respectively. If the electrodes perturb the charge distribution in the plasma to a negligible extent (i.e., if the probe distance is much larger than the Debye length) and if the plasma characteristics are similar at the electrodes, a simple expression describes the cell voltage, $U(29,30)$ :

$$
\frac{e U}{k T_{e}}=-\ln \left(\frac{I_{e 1}}{I_{e 2}}\right)+C
$$

where $C$ is constant in a given voltage range. Thus, the electron temperature is easy to determine by analyzing the voltage current data.

Electron number densities can also be recovered from current voltage curves. There is a multitude of different regimes depending on geometrical, diffusion, and flow conditions. For convection-diffusion regime, in the case of two cylindrical electrodes of unit length $(30,31)$ :

$$
n_{e}=\frac{I_{i 1}+I_{i 2}}{16 l_{p}}\left(e \mu_{i} r_{p} v_{f} k T_{e}\right)^{-1 / 2} .
$$



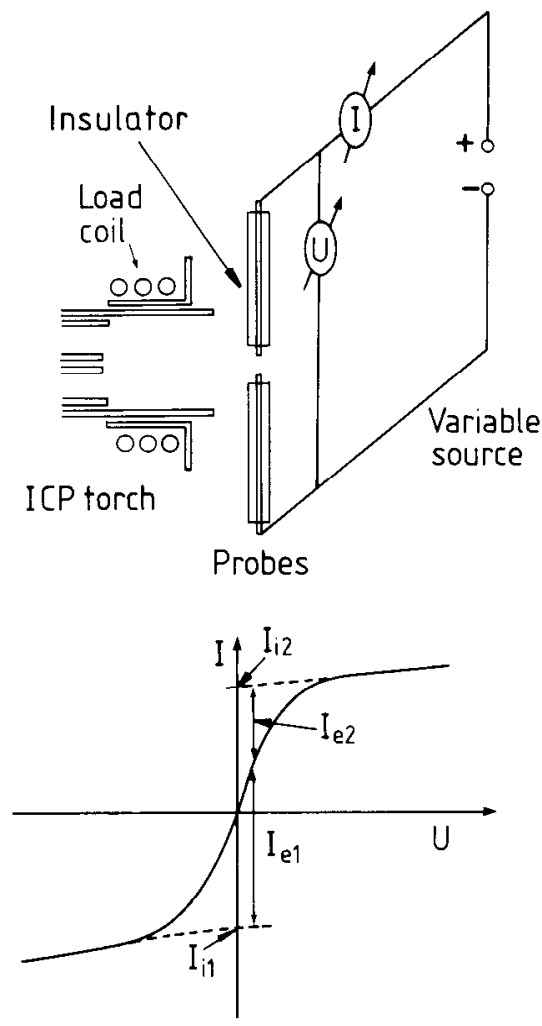

Figure 2. Two-probe electrode configuration in ICP torch plasma and typical current voltage characleristics (30).

Here $l_{p}$ and $r_{p}$ are the noninsulated length and the radius of the probes, $\mu_{i}$ is the ion mobility, and $v_{f}$ is the flow velocity of the gas at the torch axis.

Spatial dependence of the above quantities in stationary plasmas can bc dctermined by displacement of the electrode pair. The moderate resolution (at present $\sim 1 \mathrm{~mm}$ ) is determined by the physical dimensions of the probes. Transient plasma processes are hard to follow with this method because of the sluggish time response of the electrodes.

\section{B. Optical methods}

Emission spectroscopy (32-35), resonance ionization (36), and laser beam deflection on optical inhomogeneities (37) have been used in ion source plasma diagnostics.

Because the ICP emission spectrometers used in analytical chemistry are able to provide information on both electron number densities and plasma temperatures, several ways of extracting these data from the spectra were developed. Application of spectroscopic methods in diagnostics of ICP plasmas were recently thoroughly reviewed (38). Here we only quote the principles of the techniques involved in order to facilitate their application in studying other ion sources. The 
methods used are all based on the measurement of intensities, intensity ratios, or widths of optical lines. The first two of them assume an optically thin plasma; otherwise corrections are necessary for self-absorption.

If local thermal equilibrium is assumed in the plasma, the ratio of an ionic emission intensity, $I_{q p}^{+}$, toward an atomic intensity, $I_{q p}$, can be written as:

$$
\frac{I_{q p}^{+}}{I_{q p}}=2\left(\frac{2 \pi k m_{e}}{h^{2}}\right)^{3 / 2} \frac{g_{q}^{+} A_{q p}^{+} \nu_{i} T^{3 / 2}}{\mathrm{~g}_{q} A_{q p} \nu_{a} n_{e}} \exp \left[-\frac{I_{p}+E_{q p}^{+}-E_{q p}}{k T}\right],
$$

where: $A_{q p}^{+}, g_{q}^{+}, v_{i}, E_{q p}^{+}$, and $A_{q p}, g_{q}, v_{a}, E_{q p}$ are the transition probabilities between levels $q$ and $p$, the statistical weights of the levels, frequencies of the emission lines and the transition energies for the ions and the atoms, respectively, and $I_{p}$ is the ionization potential. Measuring frequencies and intensities for at least two species provides the density and temperature data if appropriate transition probabilities are available.

Measurement of continuum intensities also can provide information on the plasma. Emission related to free-free transition and recombination radiation usually dominate most of the continuum. The continuum intensity, $I_{c}(v)$, at frequency $v$ of a homogeneous, singly ionized plasma slab of thickness $d$ has the form:

$$
I_{r}(v)=\frac{16 \pi e^{2} d}{2 c^{2}\left(6 \pi m^{3} k\right)^{1 / 2}} \frac{n_{e}^{2}}{T_{e}^{1 / 2}}\left[G\left(v, T_{e}\right)+\xi\left(\nu, T_{e}\right)\left(1-\exp \left(-\frac{h v}{k T_{e}}\right)\right)\right],
$$

where: $G\left(\nu, T_{e}\right)$ denotes the Gaunt factor (25) and $\xi\left(\nu, T_{e}\right)$ is a correction factor of the recombination term accounting for the electronic structure of the atoms.

The electric field of the charged plasma particles acts on the atoms, causing Stark dispersion of energy levels and consequent broadening of the emission lines. This phenomenon provides yet another possibility to get access to information on plasma parameters. Stark broadening is most prominent on the Balmer series of hydrogen; however, it can also be observed on Ar lines between 500 and $600 \mathrm{~nm}$. For a $T_{e} \approx 10^{4} \mathrm{~K}$ plasma, FWHM of the $H_{\beta}$ hydrogen line grows from $0.05 \mathrm{~nm}$ to $5 \mathrm{~nm}$ when the electron density increases from $10^{14}$ to $10^{17} \mathrm{~cm}^{3}$. Elevating the electron temperature to $T_{e} \approx 4 \times 10^{4} \mathrm{~K}$ does not influence the relationship in this density region.

Therefore, when hydrogen is present in the system or when other elements exhibit measurable Stark broadening, this method can provide temperature-insensitive electron density data. Further possibilities-such as measuring absolute line intensities, Doppler broadening, line to continuum intensity ratio, InglisTeller method, etc.- exist to exploit emission spectroscopy in plasma diagnostics. Their application in examining mass spectrometric ion sources is moderately explored.

Spatial distributions of $n_{e}$ and $T_{e}$ are easier to recover than for electrical probes because images of sections of the plasma emission volume can be resolved spectrally and detected separately. Vertical or lateral stripes of the emission are projected to the slit of a spectrometer and a photodiode array is used for detection (34).

Resonance ionization of a selected atomic species in the plasma by tunable 


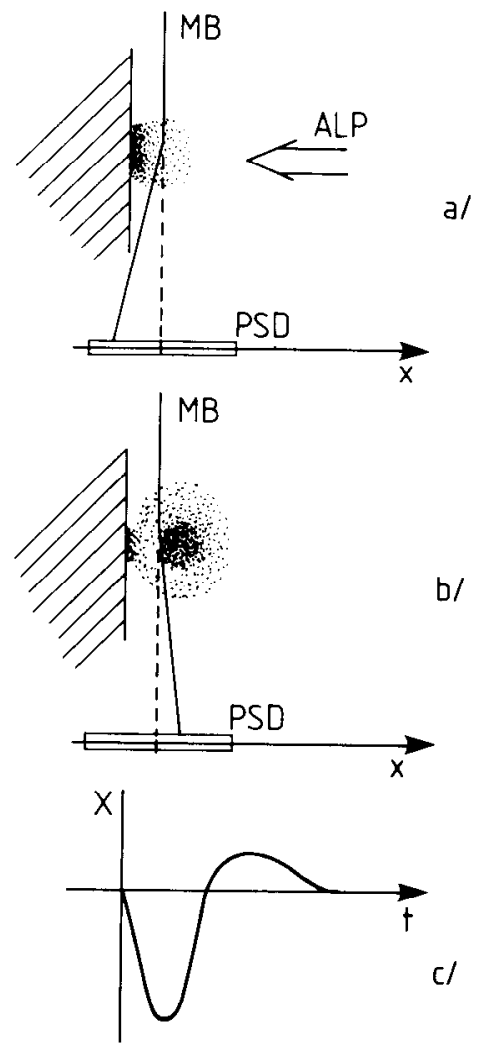

Figure 3. Deflection of monitoring laser beam on optical density gradients of expanding laser-induced plasma plume (37). ALP: ablating laser pulse; MB: monitoring beam; PSP: position-sensitive detector. (a) Early phase of expansion. (b) Plume center of gravity passed the beam. (c) Time dependence of the deflection signal.

lasers promises new possibilities. Because mass analysis and detection of the generated ions cause no extra difficulties in the case of a mass spectrometric ion source, the technique is specifically suited for this task. So far mostly analytical applications of resonance ionization mass spectrometry (RIMS) have been published. Interesting examples of combining atomization sources (glow discharge, flame) with RIMS point to the direction of diagnostic capabilities (39-42). As low as $10^{8} \mathrm{~cm}^{-3}$ number density species can be detected (36).

Transient plasmas are cumbersome to characterize for the large and quickly varying gradients of their properties. Hence, in most cases only indirect indications of their properties are available. A few investigations of laser-generated plasmas on solid/1 Torr $\mathrm{N}_{2}$ or atmospheric boundaries were carried out by fast spectroscopy or optical multichannel analyzers to record time-resolved emission spectra $(43,44)$. Postionization of laser-desorbed species was recently reported (45) and provides energy distributions for neutral particles.

Another recent development is based on the deflection of a monitoring laser 
beam by the optical inhomogeneities of the plasma. A laser ablation experiment together with the expected signal is depicted in Figure 3 (37). Optical density variations, and thus plasma expansion, can be recovered from the deflection signal.

\section{Ion extraction and analysis}

The ultimate purpose of mass spectrometric plasma ion sources is to produce ion currents with appropriate characteristics for mass analysis. To achieve this, ions must be extracted from the plasma, which is usually accomplished by the incorporation of electric fields or by entraining the ions in carrier gas flow. The extracted ions may preserve remnants of information on the plasma processes, the most important being the identity and density of ionic species in the plasma.

Transient plasmas can be created directly inside the ion source of a mass spectrometer because of their negligible load on the vacuum system. Stationary plasmas, on the other hand, need to be interfaced by using orifice, sampler and/or skimmer cones together with two or more step differential pumping in a gradual transfer of pressure to the vacuum of the mass spectrometer. Avoiding distortions of the original plasma composition in such interfaces is one of the main aims of interface design. In general, it can be achieved only on the basis of diagnosing the plasma inside the interface (46-48).

In the simplest experiment, a sample of known composition is introduced into the source, and the mass spectrum is collected. LTE-based ionization temperatures and electron number densities are determined from intensity ratios of singly and doubly charged ions of the same species or from intensity ratios of two species with different ionization potential $(49,50)$. To determine the intensity ratio for doubly and singly charged ions of the same species, the Saha-Eggert equation can be used:

$$
\frac{P^{2+}}{I^{+}}=\frac{2 P_{2}(T)}{n_{e} P_{1}(T)}\left(\frac{2 \pi k T m_{e}}{h^{2}}\right)^{3 / 2} \exp \left(-\frac{E_{2,1}-\Delta E_{2,1}}{k T}\right),
$$

where $P_{2}(T)$ and $P_{1}(T)$ are the internal partition functions for the two types of ions, $E_{2,1}$ is the ionization energy of the singly ionized particle in vacuum and $\Delta E_{2,1}$ is the depression of the ionization energy due to shielded interactions in the plasma. On the basis of the Debye-Hückel theory, this depression is frequently approximated as:

$$
\Delta E_{2,1}=\frac{2 e_{0}^{2}}{\lambda_{D}}
$$

where $\lambda_{\mathcal{D}}$ stands for the Debye length.

It is also possible to investigate matrix effects. In low-temperature equilibrium plasmas, the presence of a low ionization potential species at high density may suppress the ion signal from other species because the density of free electrons is elevated. Mass spectrometers are ideal tools to detect such effects $(51,52)$.

By analyzing standard samples, relative sensitivity factors (RSF) can be measured for a large number of elements. These factors show the combined influence of three different effects: atomization and ionization of the analyte and mass 
discrimination of the instrument (53). Therefore, conclusions on the state of the plasma based on such measurements have to be taken with care.

Ion kinetic energy measurements were conducted for ICP $(54,55)$, for spark source $(56,57)$, and in a large number for laser ionization $(58-67)$ plasmas. The methods show a large diversity including the use of simple retardation electrodes, time of flight measurements, or electrostatic anâlyzers. Nevertheless, distinctly different energy spectra for the various ions measured may throw light on differences in formation mechanisms.

It is possible to follow spatial variations of plasma inhomogeneities with mass spectrometers. The relative position of the sampler interface and the plasma can be changed with spatial resolution in a fraction of a millimeter, but optical methods are more widespread and better suited for resolving concentration distributions.

Temporal processes were investigated using mass spectrometers in the case of spark source $(68,69)$ and laser ionization $(70)$ mass spectrometry. After the instantaneous plasma generation by a single spark discharge or a laser pulse, time distributions of ion intensities were recorded. Temporal decline of the ion signal can be related to the decay of sample excitation and to changes in the state of the plasma.

\section{Mathematical modeling}

Complete theoretical description of the plasma processes should provide the spatial and temporal distributions of the density, $\rho_{m}(\mathbf{r}, t)$, velocity, $v_{m}(r, t)$ and temperature, $T_{m}(\mathbf{r}, t)$, distributions for all $m=1, \ldots, N$ components. This task is far more complex than present-day measuring and calculating capabilities can handle; thus plasma physicists have had to settle for one or two components. In the one-component case, conservation laws for mass, momentum, and energy provide a set of partial differential equations $(71,72)$ :

$$
\begin{gathered}
\frac{\partial \rho}{\partial t}+\nabla(\rho \mathbf{v})=\nabla(D \nabla \rho), \\
\rho \frac{\partial \mathbf{v}}{\partial t}+\rho(\mathbf{v} \nabla) \mathbf{v}=-\nabla p+\nabla(\mu \nabla \mathbf{v})+\sigma \mathbf{E} \times \mathbf{B}, \\
\rho \frac{\partial u}{\partial t}+\rho(\mathbf{v} \nabla) u=-p \nabla \mathbf{v}+\nabla(\kappa \nabla u)+\sigma \mathbf{E}^{2}-R+S,
\end{gathered}
$$

where $p$ and $u$ are the pressure and the internal energy of the plasma, $\mathbf{E}$ and $\mathbf{B}$ are the external electric field strength and the magnetic induction, $D, \mu, \kappa$, and $\sigma$ are the diffusivity, viscosity, thermal diffusivity, and electrical conductivity coefficients, and $R$ and $S$ are the radiation energy loss and external energy source terms.

If thermodynamic equilibrium between the components does not prevail, at least separate electron and heavy particle equations have to be established [resembling Eqs. (8)-(10)] complemented with diffusion, reaction, momentum, and energy exchange terms on the right-hand sides. Even for the one-component 
case, additional relations are necessary. Assuming that the plasma behaves like an ideal gas, we can write:

$$
p=(1+\eta) \rho k T / m,
$$

and

$$
u=\left[\frac{3}{2}(1+\eta) k T+\eta I_{p}\right] / m
$$

where $\eta$ stands for the degree of ionization. In the case of local thermodynamic equilibrium, if only singly ionized species is present, the degree of ionization can be expressed by the simplified Saha formula:

$$
\frac{\eta^{2}}{1-\eta}=\frac{m}{\rho}\left(\frac{2 \pi k T m_{e}}{h^{2}}\right)^{3 / 2} e^{-I_{p} /(k T)},
$$

The physical construction and operation of the particular ion sources determine the initial and boundary conditions for Eqs. (8)-(10). The material parameters are taken either from experiments or more frequently from statistical theory. In the case of stationary methods of ionization, the time derivatives in Eqs. (8)-(10) are set to zero, and stationary solutions are pursued.

The major differences between the various types of ion sources derive from the mechanisms of energy deposition. The MIP and laser ion sources are based on energy absorption by the sample:

$$
S=\alpha \Phi,
$$

where $\alpha$ is the absorption coefficient of the sample and $\Phi$ is the irradiance of the energy source. Glow, ICP, and spark sources energize the analyte by ohmic dissipation described by the $\sigma \mathrm{E}^{2}$ term in Eq. (10).

Solution of Eqs. (8)-(10) can be attempted by the sophisticated methods of numerical fluid dynamics, but to find the appropriate algorithm, assiduous trials of several methods are usually required (7.3).

\section{E. Miscellaneous}

Further indirect methods are in use to gain information about plasma processes in ion sources. The measurement of material consumption and surface composition modification of those parts of the electrodes which were in direct contact with the plasma can provide data on material release during the interaction (7477). Weighing the electrodes before and after exposure, scanning electron microscope (SEM) observation of the surface morphology, and surface composition determination by secondary ionization mass spectrometry (SIMS) have been applied to learn electrode behavior. Spark source and laser ionization produce craters in the sample whose depths and morphology were studied by SEM and stereopair micrography $(78,79)$. 
Cluster ions are frequently observed in the mass spectra. By isotope labeling of the sample surface, it appears possible to decide whether the clusters stem directly from sputtering or whether they are formed in the plasma itself (80-82).

In principle, any surface analytical method can be employed to investigate the interactions of the plasma with the container, with the refractory materials used for isolation, or with the sample. This is important not only for diagnostics, but also to ascertain to what extent unwanted erosion is able to contaminate the plasma and hence to distort seriously the analytical results or to lead to an undesirable background signal.

\section{ION SOURCES UTILIZING PLASMA GENERATION}

In the following subsections, we survey the plasma ion sources most widely used in mass spectrometry and the present understanding of their operation. It is obvious from the first inspection of the literature that development of the diagnostics of various sources is strongly uneven. Because of its success as an optical emission source and because of its large plasma volume, the ICP source enjoys extensive coverage. Practically all the techniques mentioned in the Section II have been assigned to understand and optimize ICP ion sources. The application of glow discharge and microwave sources is just gaining momentum; therefore, plasma diagnostics will penetrate these fields in the course of their expansion through the coming years.

Spark source mass spectrometry profited for decades from the privilege of being one of the most versatile trace analytical techniques. Unfortunately, direct investigation of its plasma was hopelessly hindered by its very short duration (on the timescale of $\sim 100 \mathrm{~ns}$ ), its small size, and its transient nature. Ion extraction studies and indirect measurements, however, are fairly well represented in the literature.

The youngest of all the reviewed sources is based on the pulsed laser solid interaction. Its plasma shares the difficulties of the spark source in duration and size. Nevertheless, considerable work has been invested in studying laser-solid interaction for its potential use in laser machining and nuclear fusion ignition by laser implosion. Ion extraction, optical and indirect methods, and mathematical modeling were used to examine the laser-generated plasma.

\section{A. Stationary regime ionization}

The ion sources listed under this subheading deliver stable ion bcams for an extended period of time; if operated properly, their stability is usually limited only by the wear and contamination of the electrodes and the refractory material. The ICP and MIP require nebulization of solutions into the plasma, whereas glow discharge is based on the direct contact of the sample with the plasma phase. Laser ablation can be used in combination with any of these methods to facilitate atomization in the glow discharge or to allow the direct analysis of solids in ICP $(40,83,84)$. 


\section{Inductively coupled plasma mass spectrometry}

Early applications of the thoroughly diagnosed (33-35,85-88) and carefully described $(71,89-93)$ inductively coupled plasma torch in mass spectrometry $(46,49)$ showed several drawbacks and neuralgic points. The very presence of the cooled metallic sampling cone in the extremely hot $(\sim 8000 \mathrm{~K})$ plasma environment was responsible for a large part of the problems encountered. Frequently, in early designs, a second discharge or "pinch" developed between this sampling cone and the first electrode of the ion optics. Also, orifice lifetime was low, and orifice clogging occurred often. Mass spectrometric peak shapes were sometimes distorted and interference from doubly charged and oxide ions of the analyte and refractory material affected the selective detection in the low-resolution (quadrupole) mass filters. Even now, when most of these problems have been diagnosed and corrected for in second-generation designs, introducing He instead of $\mathrm{Ar}$ as plasma gas leads to similar problems (94).

By means of extensive use of the plasma diagnostic practices explained in Section II, most difficulties could be traced back to two areas. By investigating gas dynamics at the sampling orifice, the presence of a boundary layer virtually at rest became apparent (47). Ions from the plasma cloud could reach the mass analyzer only after residing for several microseconds in this relatively low temperature layer where they participated in ion-molecule and recombination reactions. This "intermezzo" led to the distortion of the analytical signal. Increasing the orifice diameter from the original value of $50-75 \mu \mathrm{m}$ to $0.5-1.0 \mathrm{~mm}$ gave rise to the so-called continuum flow regime, where ions could be transported into the spectrometer by the continuous gas jet directed from the atmospheric pressure plasma towards the low pressure interface region (47). A comparison of the small aperture boundary layer sampling and the improved continuum flow sampling of ICP is shown in Figure 4. The large orifice in Figure 4(b) can be introduced only at the expense of an additional differential pumping stage and a so called skimming cone. The idea was borrowed from earlier efforts in MIP coupling to mass spectrometry (95) and molecular beam studics (96).

The increased orifice diameter and the continuum flow sampling have multifarious beneficial effects. Quite obviously, it brings about increased ion transmission and improved detection limits $(50,97)$. Orifice clogging becomes less of a problem, and oxide signals can be minimized by choosing an optimized opening diameter (50).

The other disturbing factor was the presence of a secondary or parasitic discharge. As was indicated by floating Langmuir probe measurements, large plasma potentials can be induced $(94,98,99)$, which generate the unwanted discharge to the sampling cone. The remedy for this deficiency came after realizing that the large plasma potential is a direct consequence of capacitive coupling between the load coil and the plasma (48). It can be removed almost completely by centergrounding the coil. Increased orifice lifetime, lower doubly charged and refractory oxide ion levels, and narrower ion kinetic energy distributions (affecting peak shape and mass resolution) were observed $(48,55,30)$. Comparison of impedancematching networks and the corresponding kinetic energy distributions of $\mathrm{Ar}_{2}^{+}$ ions for the two configurations is displayed in Figure 5. Furthermore, attenuation 


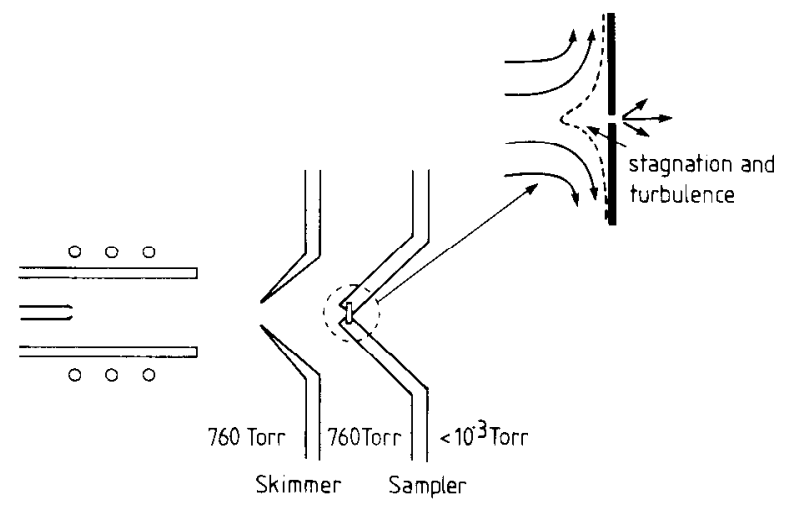

(a)

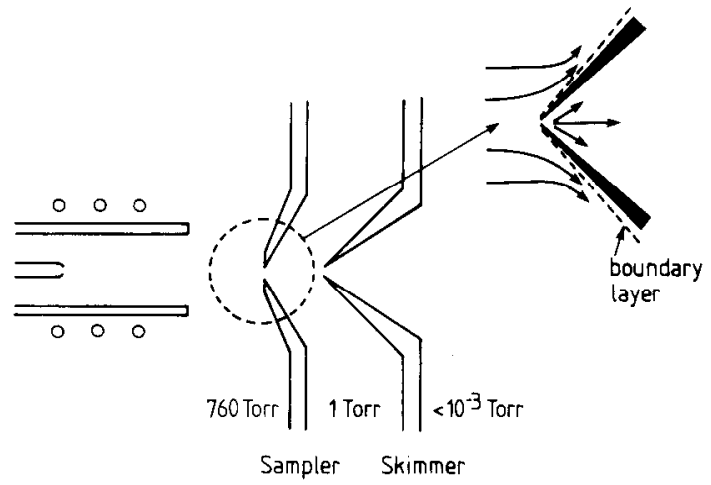

(b)

Figure 4. Changing the philosophy of plasma stream sampling in ICP-MS. (a) Boundary layer sampling. (b) Continuum flow sampling.

of radio frequency (rf) interferences between the plasma generator and the measuring electronics was achieved by careful selection of the shielding configuration $(30,98)$.

The ICP ion source has reached maturity thanks to extensive optimization of the factors influencing ion production and ion extraction. The two parameters that had the most straightforward effect on performance turn out to be the injector gas flow and the forward power of the excitation. Although optimal values (mostly in the region $0.5-1 \mathrm{~L} / \mathrm{min}$ and $1-1.5 \mathrm{~kW}$ ) depend somewhat on the instrument design and on the particular application, correlations with analyte ion signal and detection limit (100-102) and with the level of doubly charged and oxide response were established $(50,97,98,100,103)$. Without the center-ground arrangement of the load coil, artifact effects such as a strong influence of the aerosol flow rate and forward power on the kinetic energy distribution of positive ions were measured (54).

Other attempts to enhance plasma performance were less systematic, and their evaluation usually relied on a single investigation. Load coil geometry shows 


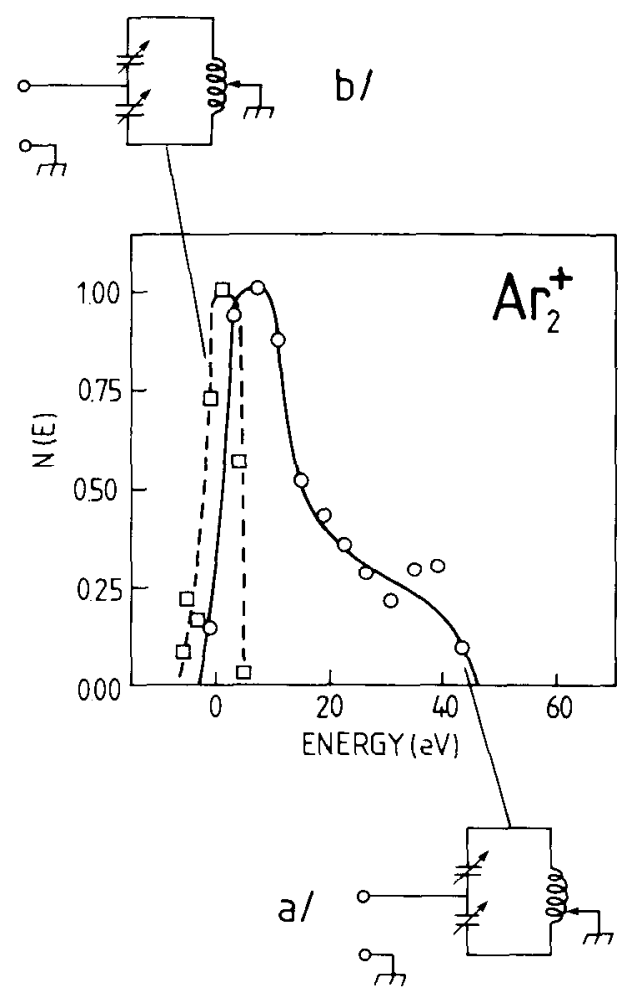

Figure 5. Changing the grounding of the load coil and its impact on the kinetic energy distribution of $\mathrm{Ar}_{2}^{+}$ions. (a) Off-center grounding of load coil. (b) Center grounding of load coil (48).

moderate influence on oxide response, on doubly charged ion yield, and on mean kinetic energy of ions $(94,103)$. For atomic emission spectroscopy, the plasma radius, $R$, was optimized as a function of operating frequency (104). $R$, and the skin depth, $s$ (i.e., the radiofrequency power penetration depth) appear to provide optimum detection limits at $R / s=2.25$. This topic was revisited recently with respect to changes in detection limit $(94,105)$. A large $R / s$ ratio has the advantage that the sample is more easily introduced and that less interference occurs between the plasma skin (where energy deposition takes place) and the axial channel (where the analyte is transported). The price of this decoupling is a lower temperature in the center, which consequently worsens detection limits.

Experiments with an $\mathrm{Ar}-\mathrm{N}_{2}$ plasma gas mixture show depression both in electron number density and ionization temperature along with the addition of $\mathrm{N}_{2}$ (106). Results of this study turn out to be useful in the understanding of energy transport from the induction region to the axial channel. Replacing the customary Ar with He in the plasma promises a higher degree of ionization (and higher sensitivity) for the less readily ionizable analyte constituents because of the considerably higher ionization potential of He $(24.6 \mathrm{eV}$ instead of $15.8 \mathrm{eV})(94,107)$. Preliminary experiments show, however, that severe parasitic discharge between the plasma and the sampling cone persists in spite of various efforts to reduce 


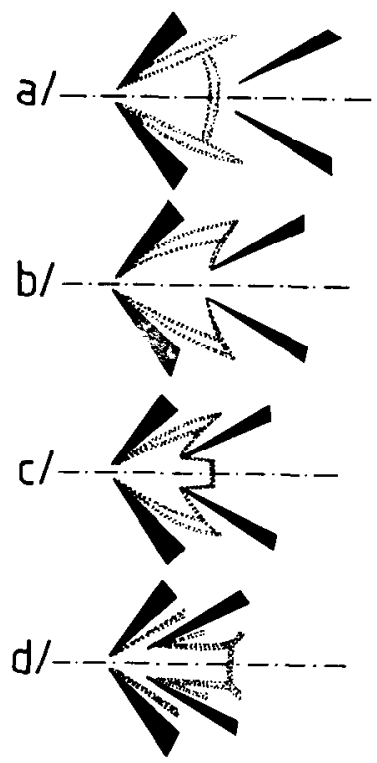

Figure 6. Scattering regions (marked by dots) for gas jet from the sampling cone at different skimmer to sampler distances (96).

or eliminate it (94). Clearly, further work is needed to find the proper operating conditions and interface design for the He ICP ion source.

Information on argon plasma temperature is available from rotational and vibrational temperature measurements and from Doppler temperatures determined from line broadening (108-110). Recently, radially resolved rotational temperatures were also measured for an ICP operated at $2 \mathrm{~kW}$ and with argon, oxygen, nitrogen, and air as outer gases (111). Spatial variations of ionization temperatures are determined by mass spectrometry using iodine as thermometric element (112).

The problems of interface geometry and sampling position were addressed several times. It was discovered that the highest unperturbed neutral beam intensity is produced if the skimmer opening position is somewhat ahead of the so-called Mach disk (i.e., the location in the expanding gas jet where the scattering of jet particles on the residual gas in the interface starts to dominate) (113). Scattering regions are marked in Figure 6 by dots for different sampler skimmer distances. The most favorable case is depicted in Figure 6(c). When the Mach disk is formed ahead [Fig. 6(a)] or at the same location [Fig. 6(b)] the skimmer cone scattering diminishes the ion intensity. In the case in Figure 6(d), the two cones are too close, and the gas load on the second stage becomes serious. The skimmer opening diameter, $D_{s}$, is determined by the requirement that the mean free path and $D_{s}$ should be closely matched to each other to achieve least disturbed jet expansion and consequently the maximum beam intensity (97). For centertapped load coil, the axial and radial positions of sampling have no significant influence on the kinetic energy of the ions, at least not within a displacement region of $10 \mathrm{~mm}$ (54). Pronounced decline in ion signal was observed for axial displacement (54) whereas, in other studies a more complex behavior was found $(100,102)$. 
Detection limits are also related to the presence of a continuum background stemming from vacuum ultraviolet photons generated in the plasma as the channeltrons used for ion detection are sensitive to this radiation. In earlier designs, off-axis positioning of the detector assisted in the prevention of false counts (46), but in later ones the introduction of an optical baffle plate in the region of ion focusing optics helps to diminish further the influence of stray radiation (102).

The conditions of sample introduction have a direct impact on the analyte plasma interaction. High concentration of the sample solution (above $1 \%$ solid content) can cause orifice clogging because of recondensation on the cooled metal surface (101). For samples with high salt content $(0.1-1 \%)$, deposition of solid matter on the sampling orifice was observed. It is possible to minimize the rate of deposition by lowering the injector gas flow and increasing rf power, sacrificing at the same time the intensity of the analyte signal (114). High matrix concentrations-especially if they contain low ionization potential species-may suppress the analyte signal by elevating the electron number density in the plasma (51), or simply by inducing space charge effects in the spectrometer (115). Finally, the effects of water vapor loading on electron number density, on excitation temperature and on the coupling between the plasma and the rf field were investigated. Whereas in the first two cases no significant influence was encountered, for the rf field coupling (measured by the reflected power) diverse results were found for different instruments (116).

In contrast to most of the other excitation sources for mass spectrometry, the ICP source has been used up to now mostly for the analysis of solutions. Direct introduction of solid samples, however, is often desirable so as to avoid lengthy dissolution, pretreatment, and the unavoidable sources of contamination during such procedures. Slurry nebulization with nebulizer types that have a wider sample supply tube than the capillary in conventional systems have been used for the analysis of different samples in atomic emission spectrometry and recently also in ICP mass spectrometry $(117,118)$. The evaporation of solid particles, especially those of refractory materials, places m:ore stringent requirements on the source. A recent study of the evaporation behavior of the slurry atomization with a Babington nebulizer and a $2.4 \mathrm{~kW} \mathrm{ICP}$ for refractory oxide powders used for the production of ceramics was published (118). It appears that the nebulization characteristics are similar to those for solution work. Electron microscopy of the aerosol collected after passing through the plasma allowed distinguishing two different particle morphologies, namely submicron condensates and partially remolten larger particles. A theoretical model describing the evaporation on the basis of axially varying temperature distribution [see measurements of rotational temperature from $\mathrm{OH}$ bands (111)] produced results comparable to those obtained experimentally.

Recent efforts in ICP diagnostics point to the direction of characterizing the plasma in the reduced pressure region between the sampling and skimming cones. Atomic emission $(119,120)$ and atomic fluorescence $(120)$, as well as Langmuir probe measurements (121) are applied to unveil the complex plasma structure in the supersonic jet.

It is evident from simply perusing the reievant journals that future prospects 
of ICP mass spectrometry are bright. The method passed the phase of development, and applications are reported in increasing numbers. Further advances in the instrumentation are being developed at present, including alternatives for sample introduction, plasma generation, and its combination with other methods of ionization (e.g., to make it directly adaptable to solids mass spectrometry as in laser ablation combined with the ICP) $(40,83,84)$. Plasma diagnostics will certainly remain an important tool for monitoring achievements and correcting trials.

\section{Microwave-induced plasma ion source}

The MIP ion source is a close relative of the common household microwave oven. The output power of a typical oven magnetron (about $500 \mathrm{~W}$ at $2450 \mathrm{MHz}$ ) coincides with the upper limit of the excitation in MIP ion sources. Quite different is, however, the power density: due to their smaller absorber volume, ion sources are exceeding ovens by 3-4 orders of magnitude in this respect. The working principle is to feed microwave power into a cavity where an absorbing mediumusually a noble gas-is flowing in a discharge tube transporting nebulized particles of the sample solution. If the absorption exceeds a certain limit, dielectric breakdown occurs. When sufficient power is supplied, the discharge becomes selfsupporting and hence capable of providing a stable pool of ions.

Similarly to the ICP, the MIP was first used in optical emission spectroscopy. The advent of atmospheric pressure Ar and especially He MIP started with an improvement in cavity design (122). Previously, stable He plasmas could only be sustained at reduced pressures. Because the He ICP was not available at that time, the promise to overtake the lead from: ICP for analytical applications-MIP seems to be running an eternal second place to $\mathrm{ICP}$ - prompted several investigations.

Construction of the new transverse magnetics (TM) cavity was based on finding a compromise between the following aims: (a) The cavity should be resonant at $v=2450 \mathrm{MHz}$. (b) Maximum power density must be achieved with the smallest feasible cavity volume. (c) The discharge tube should have a location coinciding with the position of highest electric field. The common denominator of all these requirements was found to be a cylindrical cell with diameter, $D$ :

$$
D=\frac{c(k a)_{m n}}{\pi \nu},
$$

where $(k a)_{m n}$ denotes the $n$-th root of the $m$-th order Bessel function. If the cavity is excited to the $\mathrm{TM}_{010}$ mode, Eq. (15) yields $D=93.7 \mathrm{~mm}$. The height of the cylinder is a free parameter, usually it is taken between 10 and $20 \mathrm{~mm}$. As shown in Figure 7, maximum electric field strength is obtained at the axis of the cylinder, so the quartz or alumina discharge tube was inserted at this position. Fine tuning of the cavity was realized with the insertion of tuning screws of different orientation. By using this cavity, a stable plasma of atmospheric pressure He prevailed even if the He stream was loaded with the mist of nebulized sample solution. Further improvement of the performance providing practically zero reflected power (measured at the generator) came about by the introduction of the so called 


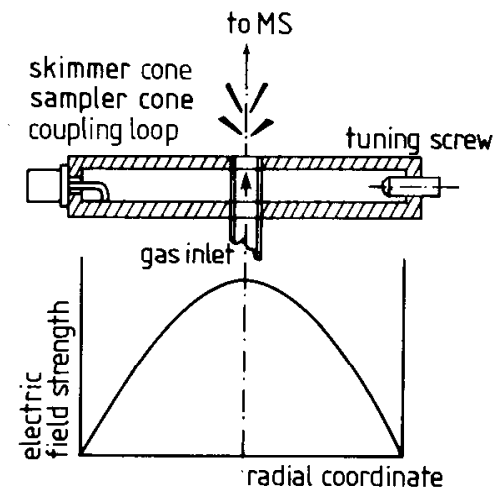

Figure 7. Cross section of the cylindrical $\mathrm{TM}_{010}$ cavity for microwave supported discharges (122). The electrical field strength is maximal on the axis, where the flow-through discharge tube is fitted. The sampling and skimming cones of the mass spectrometer interface are positioned at the exit of the discharge tube.

internal tuning (123), where the adjustment of cavity impedance was realized by tuning stubs attached to the coupling loop. Comparison of review papers of MIP optical emission spectroscopy, before (124) and after (125) the introduction of the $\mathrm{TM}_{010}$ cavity, shows the gain in performance.

Yet another finesse of MIP generation is to obtain sufficient mixing of the sample and of the dense plasma and at the same time to ensure that the plasma does not touch the wall of the discharge tube. The design of a tangential flow discharge tube solved both tasks in an elegant way $(126,127)$. In this construction, the plasma support gas entered the discharge tube close to its inner surface with a spiraling high speed flow facilitating intense mixing and resulting in a suspended discharge confined to the axial region of the tube.

Diagnostics of MIP developed, although they were less extensive than those of the ICP. Early experiments in low-pressure (0.05-2 Torr), low-power (25-50 W) plasmas indicated extremely high electron tempertures $(20,000-100,000 \mathrm{~K})$ and low electron number densities $\left(2-10 \times 10^{12} \mathrm{~cm}^{-3}\right)$. Soon after the development of the $\mathrm{TM}_{010}$ cavity, the interest, however, turned to atmospheric pressure discharges. Spatial distributions of temperatures and electron number density in $\mathrm{He}$ MIP were investigated by spectroscopic techniques (128). Variations of ionization, excitation, and rotation temperature and electron number density with the radial coordinate in the discharge tube are displayed in Figure 8 for $75 \mathrm{~W}$ forward power and $0.5 \mathrm{~L} / \mathrm{min}$ He gas flow rate. Strong differences between the various temperatures in Figure 8. point to non-LI'E mechanisms involved in the plasma formation. Potential use of this effect in the ionization of nonmetallic elements has been foreseen. Another indication of non-LTE behavior of MIP is its relative freedom from ionization interferences. Adding sodium salts to the sample in substantial concentration does not change the analyte signals (95).

Electron number densities increase with increasing forward power and little or no change with the nebulization of aqueous solutions (129). Rotational tempertures elevate slightly at lower flow rate and exhibit a weak maximum in the center of the plasma (130). Surface-wave-induced plasmas of Ar and He do not change 


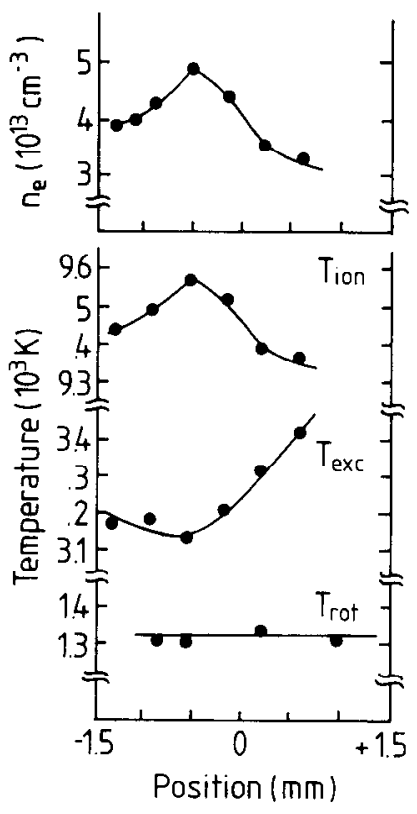

Figure 8. Radial distribution of temperatures and electron number density in atmospheric He MIP. The discharge is maintained in a tube placed in a $\mathrm{TM}_{010}$ cavity (128). Experimental parameters are given in the text.

their characteristics in the $200-2450 \mathrm{MHz}$ excitation frequency $\left(T_{\text {exc. }}(\mathrm{Ar})=2400\right.$ $\left.\mathrm{K} ; T_{\text {exc. }}(\mathrm{He})=3000 \mathrm{~K} ; T_{\text {rot. }}=2000 \mathrm{~K} ; n_{\mathrm{e}}=3-4 \times 10^{14} \mathrm{~cm}^{-3}\right)(131)$.

Originally the coupling of the MIP to a mass spectrometer marked the departure of the continuum flow sampling technique (95). The plasma extending over the open end of the discharge tube in the center of the cavity was aligned with the tip of a sampling cone as described in Section III.A.1. A quadrupole mass analyzer after the differentially pumped interface monitored the ions as a function of various experimental parameters. In its original design, the coupling turned out to be vulnerable, as air entrainment caused substantial NO formation in the plasma, leading to charge exchange reactions with ions of higher ionization potential. Similar to the situation in ICP sources, the introduction of a shield gas drastically reduces NO concentration in the plasma and eliminates the deterioration of detection limits with increasing ionization potential $(95,132)$.

Recently the interface was modified again (52). Application of a quartz bonnet to enclose the transition region between the open end of the discharge tube and the sampling cone leads to elevated temperature and a slight overpressure at this particular location. The higher temperature facilitates ionization, and the overpressure reduces further the possibility of air influx. As a consequence, the detection of halogens as positive ions became possible with reasonable detection limits (52). Optimization of sampling depth, plasma power and gas flow rates provide stable operation over a narrow range of these parameters.

Parametric studies $(133,134)$ revealed the importance of microwave forward power, gas flow rates, sampling depth, and centered sampling in determining 
ion signal and background level. Possible future trends in instrumentation are indicated in two recent reports $(135,136)$. The He MIP ion source was coupled to the output line of a gas chromatograph. Both atmospheric and low pressure plasmas prove effective in detecting halogenated organic compounds at the picogram level (135). The capabilities of MIP as a method of soft ionization were evaluated by using low pressure and surface-wave-induced excitation. Preliminary results demonstrate remarkable molecular ion intensities and the possibility of soft fragmentation by changing the forward power (136).

Possibly because of its non-LTE nature, no detailed model describing the MIP and its corresponding ion source exists, although expressions for power loss, $P_{l}$, and power absorption, $P_{a}$, are available (125):

$$
P_{l}=\left(\frac{\rho^{2} m E_{0}}{e}\right)\left(v_{i} I_{p}+\sum_{e} v_{e} I_{e}+\beta T_{e}\right),
$$

and

$$
P_{a}=\frac{1}{2} \operatorname{Re} \int_{V} \sigma|\mathrm{E}|^{2} d v
$$

$v_{i}$ and $v_{e}$ denote the frequencies of collisions leading to ionization and excitation, $I_{e}$ is the excitation energy, $\beta T_{e}$ represents the energy transported to the walls by the particles, and $\sigma$ and stands for the complex conductivity of the plasma. The symbol Re takes the real part of the complex expression. Inserting these terms in Eq. (10) and searching for stationary solutions provide some insight to the energy balance of the MIP cell at least in LTE approximation. For non-LTE treatment, however, terms describing energy conversion mechanisms between the different degrees of freedom of the system should be included.

\section{Glow discharge mass spectrometry}

There is a vast body of investigations covered in the literature concerning the glow discharge phenomenon and its utilization as a surface modification method (sputtering, etching, dcposition) and as an analytical source (optical absorption and emission spectroscopy) (137). Therefore, we will strictly confine the present review to its application in mass spectrometry and to the discussion of the most pertinent plasma physical processes involved.

It is truly justified to use the plural form if we speak about glow discharge ion sources, because more than a decade was devoted to finding appropriate geometries and working conditions for these sources (138). Hollow cathode sources were among the first tried for ionization $(139,140)$. Investigation of the radiofrequency (rf), planar diode (141), coaxial cathode (142), dual discharge (143), dualpin cathode (144), cryogenic hollow cathode (145), hollow cathode plume (146), and Grimm type (147) devices lined the path of the search for stable, efficient ionization methods of solid samples. This long list of approaches also indicates that perhaps the ultimate solution of the problem has not been found yet. At present most glow discharge instruments use a DC plasma.

Two examples of the tested constructions are depicted in Figure 9. The first 

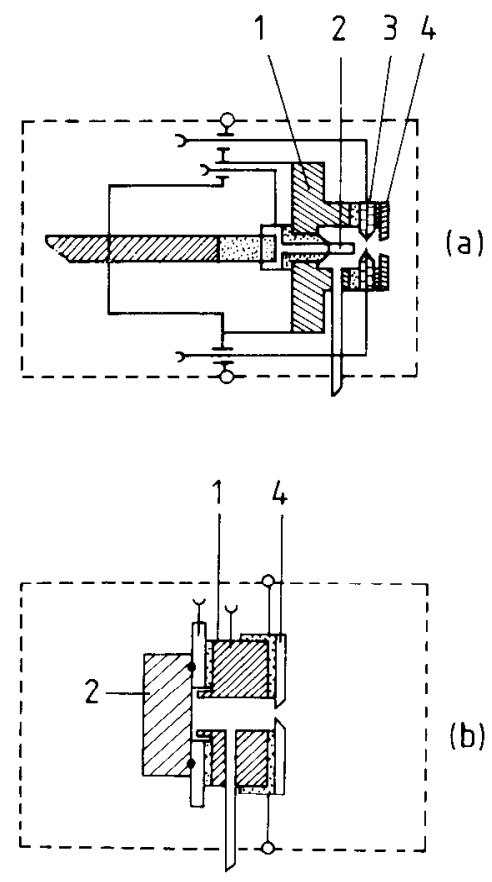

Figure 9. Two tested variants of the glow discharge ion source (147). (a) Coaxial cathode source with secondary discharge. (b) Grimm type source. (1) anode; (2) cathode/sample; (3) electrodes for secondary discharge; (4) exii aperture.

variant is a coaxial cathode source with secondary discharge, whereas the second one is a Grimm type source (147). The approximating operating conditions are shown in Table I.

According to the present understanding of these plasma devices, there are three important regions inside the source (131). Immediately in front of the cathode, there is the so-called cathode dark space. Next comes the negative glow region filling a fairly large volume of the source void. Finally, the neighborhood of the sampling orifice should be distinguished. Becausc most of the cell voltage drop is in the cathode dark space, $\mathrm{Ar}^{+}$ions generated in the negative glow are effectively sputtering the sample (connected as cathode) (149-153). Sample particles are migrating either to the surface of the walls and electrodes-giving rise to redeposition-or to the negative glow region where a fraction of them is ionized. Electron impact and Penning ionization are thought to play key roles in this process $(143,147,154-156)$. Just as in the case of other stationary plasma sources, ion sampling plays a decisive role from the point of view of mass spectrometric performance. Again, boundary layer sampling proved to be inferior to continuum flow sampling of the plasma (147).

Diagnostics of the glow discharge ion sources include measurements of sample atom populations by atomic absorption (144,157), Langmuir probe studies (147) and optical galvanic spectroscopy combined with laser depopulation of the metastable states (158). Background and analyte ion intensities for a brass sample, together with electron number densities as a function of the discharge current 


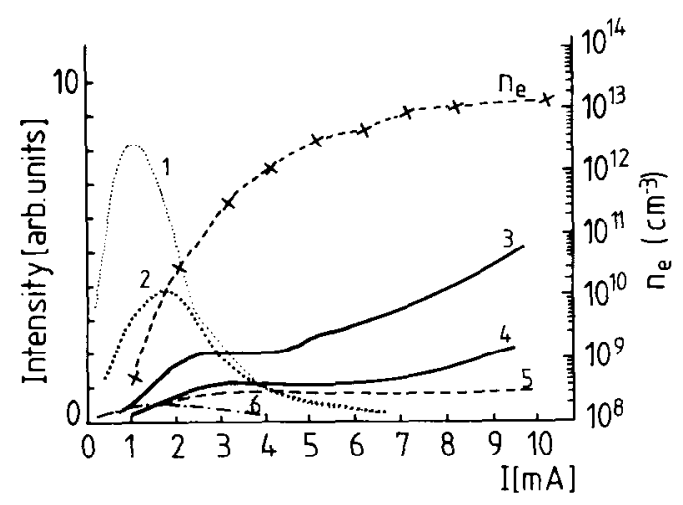

Figure 10. Background and analyte ion intensities for a brass sample, together with electron number densities measured as a function of discharge current in the cell shown in Fig. 9(b) (147). (1) $\mathrm{H}_{3} \mathrm{O}^{+}$; (2) $\mathrm{H}_{2} \mathrm{O}^{+}$; (3) ${ }^{63} \mathrm{Cu}^{+}$; (4) ${ }^{64} \mathrm{Zn}^{+}$; (5) ${ }^{36} \mathrm{Ar}^{+}$; 61 $\mathrm{N}_{2}^{+}$.

are shown in Figure 10. The electron number densities were measured by a Langmuir probe in the Grimm type source of Figure 9. It is directly apparent from this example that the potentially interfering background ion signals drop, whereas analyte signals increase with increasing discharge current, a consequence of more efficient Penning ionization and probably enhanced electron impact rates.

The factors determining analyte ion intensity turn out to be the source construction $(139-142,144,146,147,157,159-161)$, the power supplied to the source $(140,144,147,157,159,160,162)$, and the plasma gas pressure $(144,147,157,159$, $160,162)$. Removal of the molecular interferences was attempted by changing the plasma composition $(148,163)$, discriminating polyatomic ions on the basis of their different kinetic energy distributions $(146,148)$, or on the basis of their formation kinetics compared with the analyte ions by pulsing the source (148), introducing a secondary discharge $(143,147)$, and applying collision-induced dissociation in a separate cell (163).

The relative importance of electron impact and Penning ionization was also studied during searches for low interference conditions $(147,158)$. Preliminary results show a competition among these two ionization pathways and other less important channels. A high electron number density and/or a low metastable atom concentration favor electron impact processes. Ar is invariably accepted as plasma gas because of its large sputtering and ion yield and because of the relatively low level of interferences introduced $(158,160)$.

The plasma physical description of the glow discharge processes, although well developed, has not penetrated ion source applications so far. Based on stochastic description of atom, ion, and electron distribution functions, it is possible to calculate the electric field distribution, the extent of the cathode dark space, the particle energy distributions, the current densities and the sputtering yield of the 
target $(151,153,164)$. The model is based on the one-dimensional stationary Boltzmann equation (165):

$$
\frac{\partial f_{j}(x, E)}{\partial x}=z_{j} e_{0} \frac{d \Phi(x)}{d x} \frac{\partial f_{i}(x, E)}{\partial E}-\frac{f_{j}(x, E)}{\lambda_{j}}+\frac{\delta(E)}{\lambda_{j}},
$$

where $f_{j}(x, E) d E$ is the probability of finding a j-type particle at position $x$ with kinetic energy in the $(E, E+d E)$ interval. The $z_{j}$ and $\lambda_{j}$ terms denote the charge and mean free path of the j-type particles, and the $\delta(E) / \lambda_{j}$ source term describes the generation of particles having $E$ energy with the $\delta(E)$ Dirac delta function. The potential distribution, $\Phi(x)$, is cither approximated by a linear or quadratic spatial dependence or calculated in every step of the iterative solution by applying the Poisson equation (153).

A simpler approach is to start from the particle energy conservation equations [Eqs. (8) and (10)] and seek for stationary solutions. If the temperature dependence of the transport and rate coefficients is neglected, the energy equation can be decoupled and solved separately on the basis of number densities provided by the set of particle conservation equations. As a result, internal energy distribution and species concentration distributions inside the cell can then be recovered.

Future experimental possibilities include laser-assisted sputtering and ionization in combination with glow discharge $(155,156)$. The laser can serve as a tool for ablation of sample material into the glow discharge. Because lasers can be used to deposit large amounts of energy into nonconducting targets, this approach can significantly increase the versatility of the method. On the other hand, the atom populations produced by glow discharge can be ionized efficiently with tunable lasers by using the frequency of a specific atomic transition and ionizing the excited atoms with a second photon. In other words, the glow discharge can be used as stable atomization source for resonance ionization mass spectrometry (RIMS).

Recent developments of the instrumentation point toward the replacement of double focusing mass analyzers by quadrupole spectrometers in commercial instruments. The preconditions of this change are to achieve lower level of interferences from polyatomic species and to apply quadrupoles with better mass resolution. An important step in this process can be the construction of a dedicated interface (between the glow discharge cell and the mass spectrometer) on the basis of gas dynamic considerations. ICP mass spectrometry, which now routinely uses quadrupole mass filters without compromising on detection limits, is an indication that interfering species can be minimized in carefully designed ion sources and interfaces $(159,160,162)$.

\section{B. Ionization in transient mode}

To a certain extent, all the previously described stationary methods share several drawbacks. Among them we should mention the incomplete disintegration of the 
analyte particles, the low ionization efficiency, and finally, the necessity of a plasnia-maintaining medium, unavoidably leading to the formation of interfering species. Most of these weaknesses can, in principle, be eliminated by increasing the energy density, but this is not possible in the stationary regime mainly because of severe complications with the plasma confinement. Increased temperature and/or plasma particle number density will lead to the destruction of the structural elements of the instrument.

There is, however, a way to get around this difficulty by generating a hot and dense plasma for a short period of lime, by introducing the transient regime with pulsed excitation. Up to now, the two most successful transient methods have been based on the application of energy deposition by the rf spark and by the Q-switched laser. By placing the sample in vacuum and exposing it to the immense influence of a high-voltage breakdown or a giant laser pulse, it is possible to transform-at least temporarily - a microvolume of the analyte into an extremely hot and dense plasma cloud (see Fig. 1), thus providing a favorable environment for atomization and ionization. Subsequent expansion and cooling of this plasma gives rise to different elementary processes. Simultaneous ion extraction by an external field provides the first step of beam formation necessary for mass analysis.

Several factors severely hamper the diagnostics of such plasmas, among them are the limited temporal and spatial scale of the processes involved. Most deposited energy is delivered within 10-100 ns in the case of the rf spark and within $5-50 \mathrm{~ns}$ in the case of laser excitation. The spatial domain of interest falls in the region of 1--100 $\mu \mathrm{m}$. Another source of concern is the huge power density concentrated into this microvolume, leading to a strong nonstationary behavior and to an anomalous structure of the solid surface of the atomized species.

Only a few of the previously discussed measuring techniques can cope with such conditions, and even those are limited to trace postexcitation events on the $\mu \mathrm{s}$ time scale. Deflection of a laser beam on optical inhomogeneities of a plume ablated from a Si surface by a pulsed laser (37) or the application of the anomalous dispersion technique (166) to study number density distributions of postdischarge species are examples of specific studies which can be mentioned here. More common, however is the application of the ion extraction method and of other indirect methods as will be demonstrated in the next section.

The theoretical treatment of transient plasmas is bound to be subject to serious simplifications. The most important among them is probably the need to assume decoupling of energy deposition, atomization, ionization, plasma expansion, and recombination processes. In reality, all of these substeps are proceeding simultaneously, but to avoid the impossible task of solving coupled sets of partial differential equations [similar to Eqs. (8-10) for each species], the different time stages of the interaction are assigned to the dominant process during that particular stage. In this sense, it is possible to separate, for example, plasma heating and expansion, as in the so-called adiabatic absorption model (167).

In the following subsections, we will visit the recent achievements of spark source and laser ionization mass spectrometry in particular the plasma physical understanding of their fundamental aspects. 


\section{Spark ion source}

Before the conception of lasers, sparks were the ultimate sources of light pulses, unparalleled in sort duration and high intensity (168). Their applications ranged from high-speed photography (capable of framing bullet motion) to the generation of X-ray, electron, and ion bursts. Even nowadays, lasers are pumped by means of flashes stemming from electrical breakdowns.

Diagnostics of atmospheric and high-pressure sparks are ahead of vacuum spark investigations. For example, it has been possible to recover spectral opacity profiles and absorption coefficients in spark channels with $20 \mathrm{~ns}$ time resolution using a Kerr cell shutter combined with a spectrograph (169). With the introduction of picosecond lasers, the possibilities to discover the fine structure of spark generation and development, in principle, became even better. In mass spectrometry, where vacuum sparks are utilized for ion generation, diagnostic possibilities are far from being fully established or even outlined.

Two excellent reviews of spark source mass spectrometry recently appeared $(170,171)$, both of which cover the fundamental aspects of vacuum spark discharge as well. The overwhelming majority of spark source investigations were carried out in the pulsed rf spark operation mode. 'This ion source is quite simple, consisting of two electrodes with controllable separation placed in vacuum. With pulse trains of $1 \mathrm{MHz}$ rf high voltage (up to $100 \mathrm{kV}$ ), the electrodes are driven repeatedly through the different phases of the sparking process. According to the established classification, four separate stages of the sparking process are distinguished $(172,173)$.

1. Prebreakdown stage. During this $100-250$ ns period, the voltage grows on the electrodes, and a low (0.1-1 mA) accompanying current can be observed through the gap. The current is mainly due to field emission of particles from microprotrusions on the electrode surface.

2. Breakdown initiation stage. Reaching the breakdown voltage, avalanche-like processes are initiated, leading to the takeover of other mechanisms. Probably the best definition of this stage identifies it with the period between the plasma ignition and formation of the "backbone" of plasma filament in the interelectrode gap. Its duration is estimated in the $0.5-10$ ns range.

3. Spark stage. In the spark phase, the current increases irreversibly up to 10-30 A. The heavy sputtering of anode material is due to the bombarding electrons and leads to a sharp increase of the plasma volume in the gap. The length of this phase in commercial SSMS instruments is $0.5-3.0 \mu \mathrm{s}$, depending largely on the discharge circuit.

4. Arc stage. If the spark power supply is capable of delivering the necessary current, the transient processes are damping out, and a stationary state is reached. The structure of the arc plasma resembles that of the glow discharge cell with substantially elevated particle number densities (see Sect. III.A.3). Just as in the case of glow discharge, the plasma is maintained via sputtering of the cathode by the ions accelerated in 
the potential buildup in front of it. In most commercial mass spectrometers, the sparking process is interrupted before reaching this stage. The characteristics of the low voltage discharge were described in Ref. 174 .

Strictly speaking there is no plasma state involved in the prebreakdown stage. Its diagnostics, however, are well devcloped, and the applied methods resemble in many respects those of the plasmas. Experimentally, the prebreakdown stage can easily be separated from the other stages because of its reversibility. Therefore, most results have been obtained under DC circumstances.

Early investigations have shown that the breakdown equation relating the gap width, $g$, and the breakdown voltage, $U_{b r}$, has the form:

$$
U_{b r}=C_{g}^{\gamma} \text {. }
$$

where $0.6 \leqslant \gamma \leqslant 0.7$ and $C$ is a material and configuration-dependent factor (175). Measurements of prebreakdown currents and neutral atom densities by spectroscopic methods make it clear that stationary field emission or the bombarding effect of the electron beam directed from the cathode towards the anode are insufficient to initiate the breakdown (176).

Later the role of the vacuum system was emphasized in field emission current and corresponding $X$-ray measurements. In an ion-pumped system at $10^{-10}$ Torr field emission prevailed, but, at $10^{-6}$ Torr (achieved by oil-diffusion pump), enhancement of the prebreakdown current by a factor of up to $10^{4}$ was observed (177). Identification of the charge carriers was possible by time of flight mass spectrometry. The measurement confirmed the contribution of ions from both the electrodes and the residual gas (178).

Anticipation of a sudden change leading to the breakdown was based on electric current and optical absorption measurements (179). According to the so-called drop model, the breakdown initiation is the "avalanche amplification of current in electrode vapor generated by the evaporation of an anode macroparticle during its transit to the cathode" $(180)$. More complex mechanisms involving the heating of both electrodes by particle fluxes were discussed in several papers (181-184). Consensus on the details of the breakdown initiation stage has not yet been reached, and largely because of its short duration and the sporadic application of appropriate diagnostic techniques.

From a thcoretical point of view, it is tempting to associate the formation of a plasma "backbone" in the gap with a wide family of percolation phenomena. It has been shown that in the mixture of conducting and nonconducting volume elements, there exists a critical volume fraction of conducting subsystems where a dramatic change in the conductivity of the whole system takes place (185).

Largely increased volumes of material and energy transport are related to the spark stage. Plasma formation is facilitated by the self-amplifying mechanisms involved. Increasing the current leads to plasma heating and more intense sputtering of the electrodes, which in turn generate more and more charge carriers amplifying the current. This stage is most suited for diagnostic observations, although most work is only related to the registration of postspark consequences. 
According to energy conservation considerations [similar to those expressed in Eq. (10)], the deposited energy in the system is transformed into energy forms related to evaporation, atomization, ionization, hydrodynamic flow, and radiation.

Atomization has a slightly different meaning from evaporation because it incorporates the rupture of not only the bonds in the solid but cluster and molecular bonds as well. For elemental analysis, it is clearly favorable to disperse the material in the form of atoms. In the case of metals and semiconductors, the first ionization potential and atomization energy, $E_{a t}$, are comparable quantities $(72): I_{p}^{\mathrm{Na}} / E_{a t}^{\mathrm{Na}} \approx$ $4.9, I_{p}^{\mathrm{Fe}} / E_{a t}^{\mathrm{Fe}} \approx 2.15, I_{p}^{\mathrm{Si}} / E_{a t}^{\mathrm{Si}} \approx 4.6$ and $I_{p}^{\mathrm{Ge}} / E_{a t}^{\mathrm{Ge}} \approx 2.7$. Therefore, it is surprising that according to estimations only about $1 \%$ of the total energy in the spark is spent for atomization $(181,172)$. Time-resolved measurements of ion production yielded evidence that different types of ions are formed during different phases of the spark. The ascending order of formation times is: multiply charged atomic, singly charged atomic, singly charged cluster, and molecular ions $(68,69)$. The relative sensitivity factors showed strong correlation with atomization energy (173). Rf (186), and more convincingly, DC spark experiments (75) are in accord with the so-called anode mechanism (i.e., it is the anode that is primarily eroded).

The fate of the eroded material is not only evaporation and atomization. Considerable amounts of molten material exit in the form of macroparticles or droplets and stick to nearby surfaces $(76,186-191)$. Their composition differs from that of the electrode, and the maximum size of the particles is typically several micrometers.

The surface left behind after sparking has also been studied. Scanning electron microscope observation disclosed the presence of molten material on the surface $(75,76,187)$. Modification of surface composition during sparking was demonstrated by SIMS in-depth analysis $(74,77)$. Electron probe microanalysis of used clectrode cross sections showed a few $\mu \mathrm{m}$ deep homogeneous molten layer. The study of spark craters also indicates that the electrode material melts and evaporates before it enters the interelectrode gap. The relation between the crater volume and crater radius and the melting point of the electrode material was confirmed by a number of authors $(76,181,183,184)$. Relative sensitivity factors appear also to be related to the boiling point of the sparked material and its gasphase heat of formation (192), suggesting the important role of fractionation during evaporation.

During the plasma phase, the most important processes are the ionization, the expansion, and the recombination. Assuming that the amount of material entering the plasma is proportional to the amount of eroded material, we can expect a similar relationship as was found between the sparked-off volume and the melting point. In Figure 11, the pronounced decay of crater volume with increasing melting point is demonstrated.

At the peak of the energy deposition the plasma is dense $\left(10^{18}-10^{19} \mathrm{~cm}^{-3}\right)$, very hot $\left(\sim 2 \times 10^{5} \mathrm{~K}\right.$ temperature is estimated considering LTE), and strongly ionized $(172,68,69,192,193)$. Subsequent cooling, expansion, and recombination give rise predominantly to singly charged species and cluster formation $(173,192)$. Diagnostics of these processes has been scarcely performed, and we mainly rely on 


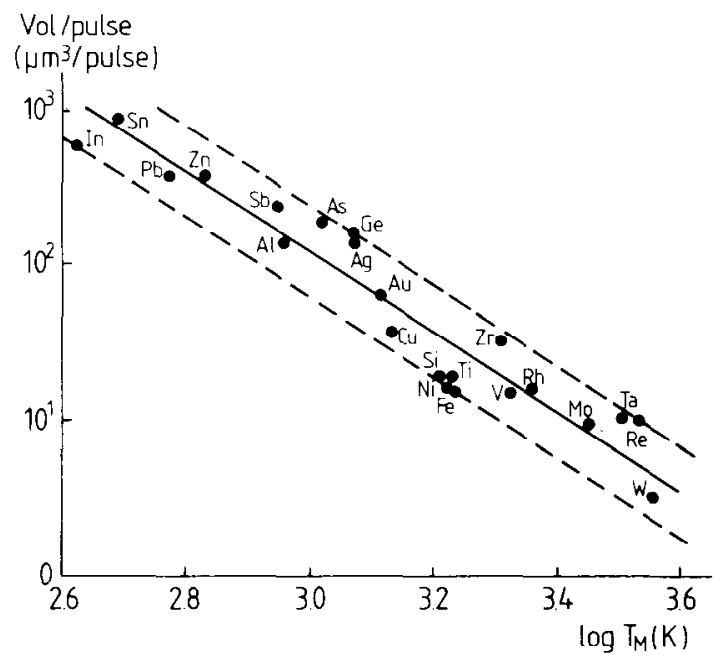

Figure 11. Volume of electrode material consumed in a single pulse as a function of the melting point of the electrode. The spark parameters were fixed: $U_{b r}=3.2$ $\mathrm{kV}$; repetition frequency $=3 \mathrm{kHz} ; \tau_{\text {pulse }}=20 \mu \mathrm{s}$. The solid line represents the least square fit whereas the dashed line stands for the factor 2 margin (76).

time-integrated and averaged optical (188) and X-ray (194) spectra. Temporal and spatial variations of species densities emerged from recent anomalous dispersion measurements (166). Figure 12 shows spatial distributions of $\mathrm{Na}$ atom number densities arising from the sparking of a $16 \%$ Na-graphite pellet $40 \mu \mathrm{s}$ after breakdown initiation (166). Inspection of this figure supports an unusual view of the spark plasma development. The postdischarge sodium atoms occupy a toroidal spatial region, rather than a sphere, as is obvious from the off-axis maximum of the concentration profiles. This expanding toroidal plasma is in contrast with the conventional picture based on spherical symmetry.

Ion kinetic energies were found to be in the range of $100-1000 \mathrm{eV}(57,195)$ : A hydrodynamic mechanism or the internal ficld induced by the escape of the lighter, and consequently, faster electrons can account at least qualitatively for these extremely high energies. Other authors explain the high ion energies by oscillations in the effective accelerating field experienced by the ions (196).

The theoretical characterization of the sparking process has been mainly devoted to the estimation of relative sensitivity factors. These are routinely used to correct the analytical results in spark source mass spectrometry, and their firm determination straightforwardly influences the accuracy of the method. Direct correlation between the relative sensitivity factors and atomization energy, ionization potential, boiling point, bond strength, vapor pressure, and plasma temperature were described $(173,192,197,198)$. Most probably the interplay of several of these factors together with instrumental discrimination effects depending on the spark conditions (geometry, electrode temperature, etc.) and ion extraction (199) can account for the RSF variations. Practically all approaches to obtain con- 


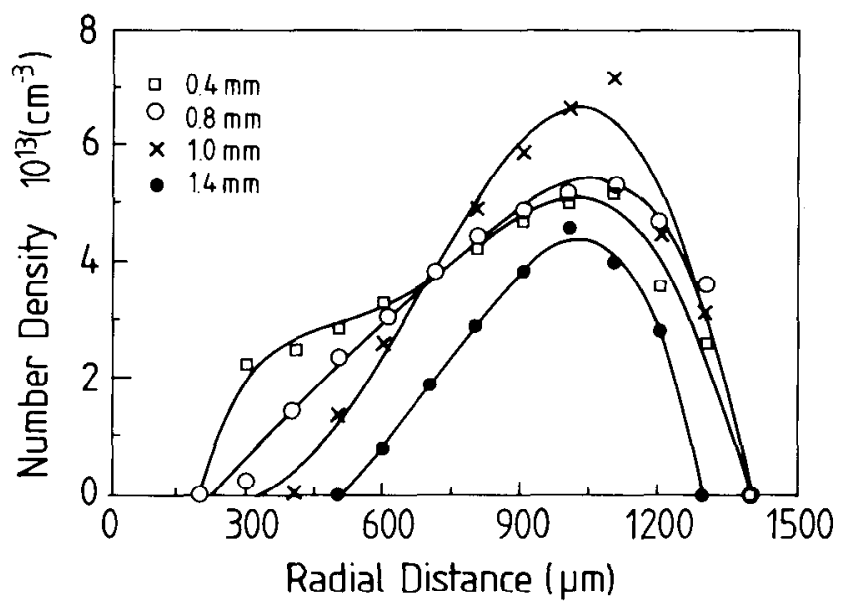

Figure 12. Na atom number density distributions $40 \mu$ s after the spark breakdown measured by the anomalous dispersion technique. The different symbols correspond to different elevations above the cathode (166).

sistency in the data have been based on meeting local thermal equilibrium conditions in the plasma and the applicability of a Saha type formula [see e.g., Eq. (13)]. These premises are questionable in themselves, as is apparent from the necessity of introducing arbitrary parameters in the procedure of fitting final equations to the experimental data.

More basic work is needed, and it should include quantitative accounting of material, momentum, and energy transport processes during the different phases of the spark. Nonstationary solutions of Eqs. (8)-(10) are difficult to find because of the immense and abrupt changes in the excitation provided by the Ohmic dissipation. If the electrodes are placed on the same axis, the problem can be reduced to two dimensions because of the cylindrical symmetry. Anomalous hydrodynamic phenomena (such as shockwave formation in the plasma and compression waves in the electrodes due to the vigorous energy deposition) are also expected. The presence of a large backpressure of the leaving vapor on the electrode surface is indicated by the splashing of the molten electrode material in the form of droplets as evidenced in several studies $(183,184)$.

Future plasma diagnostic investigations and attempts to improve the overall analytical characteristics of spark source mass spectrometry might be discouraged by the gradually descending popularity of the method and by the shrinking market share of spark source mass spectrometers. However, predictions should be cautious because SSMS has already produced a remarkable comeback in the early 1980s. Perhaps more fundamental understanding and firmer control of the sparking process could improve the reproducibility and decrease the analysis time of spark source mass spectrometry (200). Simultaneous advances in position sensitive ion detectors could give rise to a new instrument with interesting characteristics for multielement panoramic ultratrace analysis. 


\section{Laser plasma ionization}

Laser ionization mass spectrometry became a method of great significance during the seventies with the spread of Q-switched lasers. Q-switching continues to prevail as the best way of delivering the appropriate amount of laser energy within a 5-50 ns time interval. This short impulse operation is quite well fitted to time of flight (TOF) mass analyzers and even makes the conventional pulsing of TOF sources unnecessary. At high laser irradiances, compensation for the large kinetic energy spread of the generated ions is unavoidable. In most cases, the secondorder energy focusing provided by a single-stage reflector is sufficient. By way of contrast, in double-focusing instruments, signals from many repeated shots need to be integrated.

In principle, the construction of laser sources is very simple. The sample is placed in vacuum in the form of a thin film or as a bulk specimen, and the laser light is focused on the surface at the ion optical axis. Emerging ions are extracted by an electrostatic field. This structural ease combines with large flexibility provided by the continuously growing choice of lasers and with the versatility of changing ionization regimes by changing irradiance.

It is this diversity, however, that makes the subject so complex and hinders straightforward classification. To avoid misinterpretation, we ought to distinguish in every particular experiment among:

Laser types $\left(\mathrm{CO}_{2}, \mathrm{Nd}-\mathrm{YAG}\right.$, ruby, $\mathrm{N}_{2}, 4 \omega \mathrm{Nd}-\mathrm{YAG}$, dye, excimer)

Irradiances $\left(10^{5}-10^{11} \mathrm{~W} / \mathrm{cm}^{2}\right)$

Target types (inorganic: metal, semiconductor, insulator; organic; opaque; transparent)

Geometry of excitation and extraction (transmission, reflection)

The most pronounced distinction is based on the level of the irradiance, or more precisely, on the deposited energy density in a single pulse: $\left(1-R_{0}\right) \Phi \alpha \tau_{\text {pulse }}$ where $\tau_{\text {pulse }}$ is the pulse length of the laser shot and $R_{0}$ is the reflection coefficient of the material; $\alpha$ and $\Phi$ have been defined earlier [Eq. (14)]. Assuming moderately reflecting opaque targets, we can translate the distinctions into irradiance differences because of the uniform pulse length of Q-switched lasers (Vertes, A.; Juhasz, P.; Balazs, L.; Gijbels, R., manuscript in preparation).

Low irradiance $\left(\approx 10^{5}-10^{6} \mathrm{~W} / \mathrm{cm}^{2}\right)$ : Physical processes are dominated by surface heating and thermal or nonthermal ionization of target particle.s. Mass transfer across the solid vacuum interface is low. The mass spectrum contains a significant amount of molecular ions. This regime is favored in molecular weight determination of organic molecules.

Medium irradiance $\left(\approx 10^{7}-10^{8} \mathrm{~W} / \mathrm{cm}^{2}\right)$ : Evaporation of the sample becomes nonnegligible. Laser energy is absorbed both by the ionized vapor in front of the target and by the target itself. In this case complicated fragmentation and recombination processes and/or ion-molecule reactions take place in the expanding plume. The mass spectra may provide structural information for organic samples.

High irradiance $\left(\approx 10^{9}-10^{11} \mathrm{~W} / \mathrm{cm}^{2}\right)$ : The deposition of laser energy is governed 
by the strong absorption of the expanding plasma cloud created in the early phase of the laser pulse. Elemental lines are abundant in the mass spectra, providing fair possibilities for trace analysis of inorganic solids.

Although extremely important work is going on in the field of laser desorption [see a recent review (201)] involving low irradiances, we will confine the discussion to higher power densities where the concept of plasma as part of the ionization process and, therefore, the methods of plasma diagnostics are more justified. The area was surveyed in two excellent reviews some time ago $(78,202)$.

According to the energy deposition concept, different stages and regions are identified for the laser-target interaction (72). Naturally, the complete spatial and temporal separation of the involved processes is not possible because they are proceeding simultaneously. In each region and phase, however, the most characteristic component can be emphasized.

a. Induction phase: The incoming photon flux excites the internal degrees of freedom of the material. Depending on the laser frequency and on the type of target material, the deposited energy transfers into intramolecular or lattice vibrations or electronic excitation of individual particles or to the collective electrons of the solid. During this phase, sharp changes in the optical and thermal properties of the material are frequently encountered, leading to strongly nonlinear effects, to enhanced absorption and heating, and eventually to phase transitions. This stage is also characterized by the lack of convective transport. Therefore, energy transfer is more important than mass transfer.

$b$. Redistribution phase: The strongly excited volume of the sample is subject to vigorous redistribution processes. Heating, structural deformation, target erosion, and ionization take place together with interaction of the central and the peripherai parts of the irradiated spot. If, for example, the TEM $\mathrm{T}_{00}$ mode of the laser is used, a Gaussian radial distribution of the irradiance can be observed. Thus, the center of the spot is heated to a much higher temperature than the rim. Photons, electrons, ions, and neutrals are emerging from the interaction region in large quantity. Massive material flow perpendicular to the surface and turbulent intermixing in radial direction are present. In some cases, the back pressure of the departing gas leads to radial flow of the molten surface layer, manifesting itself in splashing and material buildup on the rim of the spot. If the number density of the electrons in the plume reaches a certain value, the absorption of the plasma may exceed the normal absorption leading to strong heating of the electron gas. This process is self-amplifying because the heating in turn will produce more electrons capable of further absorption. Nonlinear plasma behavior, however, would contribute only above $10^{12} \mathrm{~W} / \mathrm{cm}^{2}$ irradiance (72).

c. Free expansion phase: After the climax of the laser pulse, less and less energy is pumped into the interaction region, consequently cooling and expansion of the plume become dominant. In the cooling and expanding environment, recombination and ion-neutral reactions give rise to radiation and to new type of species. In this phase, due to the falling electron number density, the Debye screening length is growing, making external field penetration increasingly possible (72). If earlier, a dense plasma has been formed (as it is the case at high irradiances), this phase provides the first possibility of ion extraction. The outgoing ions can 
be produced from atoms, molecules and their fragments or from clusters in singly or multiply charged forms.

Separate investigations of the induction phase have not been carried out, probably because of the extreme space and time resolution demands it would impose on detection (data collection from a $10^{-3} \mathrm{~mm}^{3}$ volume with better than several ns time response). In a certain way, however, the laser desorption experiments can be regarded as if the irradiation was interrupted at this stage. In the overwhelming majority of cases, the diagnostic method is the mass and energy analysis of the desorbed ions and neutrals.

The question addressed in many papers-whether the initial energy deposition involves thermal or nonthermal processes-very likely is to be resolved along the lines of distinguishing the lasers and the samples under consideration. As a rule of thumb, infrared lasers lead to intramolecular vibrational excitation and to photon generation $(62,63,201,203)$, whereas ultraviolet wavelengths induce intramolecular electronic transitions, exciton generation and transitions between the valence and conduction bands (204-208). Eventually, in certain materials, the electronic excitation can decay very fast into vibrational modes (i.e., the nonthermal mechanism can transform by itself into a thermal one) $(206,207)$. The temperature rise induced by a laser beam on solid surfaces can be determined in analytical form by solving Eq. (10) in the $\mathbf{v}(r, t) \equiv \mathbf{O}$ case for constant $(209,210)$ and for variable (211) thermal conductivity.

The energized surface layers emit particles in large number and variety. Departure of neutral particles takes place earlier $(212,213)$, and their number certainly reaches larger proportions $(205,208)$ than that of the ions. Ion formation, if it is not thermal, is usually altribuled either to desorption of ions preformed on the surface or to photoionization of desorbed neutrals $(201,204,207,214,215)$.

During the second stage of the laser target interaction, while further energy is devoted to proceed along the primary excitation pathway, secondary processes start to occur. Further heating of the surface results in sublimation, melting and other possibly nonequilibrium phase transitions, which in turn dramatically increase the desorption rate. Thus, the developing plume in front of the target absorbs more and more laser light, thereby screening the surface from further heating.

The history of the plume depends largely on its chemical composition and on the inherent inhomogeneities in it. If the laser irradiation continues, the partially ionized plume will exhibit plasma absorption [i.e., plasma oscillations become excited (216)]. Whenever the plasma absorption reaches the normal absorption of the gas cloud, the self-amplifying mechanism mentioned under entry $b$ is invoked. Above this, so-called plasma ignition threshold (167), the temperature rises dramatically, and multiply charged ions become abundant $(58,59,217-219)$. The presence of this plasma is also proved by the presence of its continuous light emission spectrum $(43,44)$. The initial broad continuum spans over the $250-550$ $\mathrm{nm}$ region and, after 20-100 ns, gives rise to sample-specific line spectra.

On the other hand, if the irradiance of the laser pulse is just enough to approach the real plasma absorption regime, the atomization remains incomplete, and ionmolecule reactions $(220)$ as well as cluster formation are detected $(81,221,222)$. 


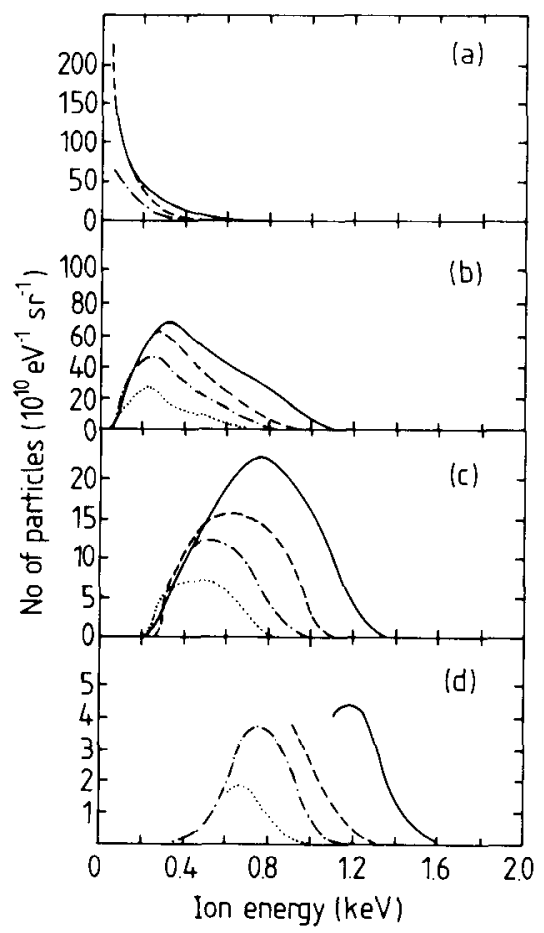

Figure 13. Kinetic energy spectrum of (a) $\mathrm{Ta}^{+}$, (b) $\mathrm{Ta}^{2+}$, (c) $\mathrm{Ta}^{3+}$, and (d) $\mathrm{Ta}^{4+}$ ions at normal incidence of Nd-YAG laser pulse $\left(\approx 10^{10} \mathrm{~W} / \mathrm{cm}^{3}\right)$ on tantalum target. Detection angles are $20^{\circ}(-), 30^{\circ}(---), 45^{\circ}(-.-)$ and $60^{\circ}(\ldots \ldots)(226)$.

The remarkable presence of cluster ions can also be deduced from direct ionization of the corresponding neutral particles on the sample surface, but isotope-labeling experiments support the gas-phase recombination mechanism (81). Analyses of cluster size distributions as a function of laser irradiance indicate that low power densities facilitate cluster building, whereas medium and high irradiances promote cluster decay (223).

Although calculations show that the temperature can rise to $10^{5} \mathrm{~K}$ in laser ionization experiments $(72,224)$, multiply charged ions are rarely detected below $10^{10}$ $\mathrm{W} / \mathrm{cm}^{2}$ irradiance. This contradiction points to the possibility of extensive recombination during the expansion phase of the interaction. Kinetic energy spectra of multiply charged ions show that the low energy part of the distributions disappears with increasing degree of ionization $(218,226)$.

Angle-resolved kinetic energy distributions of $\mathrm{Ta}^{+}, \mathrm{Ta}^{2+}, \mathrm{Ta}^{3+}$, and $\mathrm{Ta}^{4+}$ ions are displayed in Figure 13. Forward-directed high-energy ion generation and upward shifting of the peak of the spectra with increasing charge are pronounced. Therefore, in contrast to the spark plasma evolution, the plume resembles a distorted sphere, rather than a torus. The decay of the low energy part of the spectra with increasing number of charge is widely interpreted as an evidence for recombination processes. In another study, the fastest ion velocity and the maximum ionization stage scales as the power of target atomic number and show 
characteristic bend at large atomic numbers (227). The influence of recombination has also been justified in these extensive experiments utilizing both Q-switched $\left(\tau_{\text {pulse }}=20 \mathrm{~ns}\right)$ and mode locked $\left(\tau_{\text {pulse }}=100 \mathrm{ps}\right.$ and $\left.30 \mathrm{ps}\right)$ lasers. These results, indeed, leave little doubt about the importance of recombination in the expanding plume.

In reality, a vast number of other elementary processes are generated in the plasma. These can all be characterized by rate equations and corresponding time constants. Classes of these processes are the following.

Radiative excitation:

$$
A(q)+h v \rightarrow A(q+r)
$$

Significant transition probably exists only under resonance conditions.

Collisional excitation:

$$
A(q)+e \rightarrow A(q+r)+e .
$$

Radiative ionization:

$$
A^{z+}+h \nu \rightarrow A^{(z+1)+}+e .
$$

Collisional ionization:

$$
A^{z+}+e \rightarrow A^{(z+1)+}+2 e
$$

Fragmentation:

$$
A B+e \rightarrow A^{+}+B+2 e .
$$

These reactions are energy consuming; therefore, most are favored during the induction and redistribution periods. The corresponding opposite processes become important in the expansion phase.

Radiative decay:

$$
A(q+r) \rightarrow A(q)+h v
$$

Collisional decay:

$$
A(q+r)+e \rightarrow A(q)+e
$$

Radiative recombination:

$$
A^{(z+1)+}+e \rightarrow A^{z+}+h v
$$

Three-body recombination:

$$
A^{(z+1)+}+2 e \rightarrow A^{z+}+e .
$$

Ion-molecule reactions:

$$
A^{z+}+B C \rightarrow A C^{(z-1)+}+B^{+} .
$$

The number of possibilities is enlarged further by considering negative ions and surface-assisted processes.

The rate equations are built up of terms characterizing the elementary processes. 
Thus, for example, if all the collision rates are negligible, a two-step photoionization can be described by:

$$
\begin{gathered}
\frac{d n_{q+r}}{d t}=\sigma_{1} \frac{\Phi}{h v_{l}} n_{q}-\left(\sigma_{2}+\sigma_{3}\right) \frac{\Phi}{h v_{l}} n_{q+r}-k_{r d} n_{q+r} \\
\frac{d n_{i}}{d t}=\sigma_{3} \frac{\Phi}{h v_{l}} n_{q+r},
\end{gathered}
$$

where $n_{q}, n_{q+r}$, and $n_{i}$ are the number densities of the particles in the ground, excited, and ionized states, respectively. $\sigma_{1}, \sigma_{2}$, and $\sigma_{3}$ are the cross sections of the radiative excitation, stimulated emission, and radiative ionization, whereas $k_{r d}$ stands for the radiative decay rate coefficient. $\nu_{l}$ denotes the wavelength of the laser. Solution of these type of equations should be sought in combination with Eqs. (8)-(10), which makes uncompromised treatment hopeless. With the exclusion of convection, however, preliminary attempts have been made (201).

The expansion and the reactions do not stop at the end of the laser pulse. Different methods, including spatially and temporally resolved absorption $(228,229)$, scattering (230), and refraction (37) of a probing laser beam were utilized in mapping the concentration distributions of different species. Improving the resolution of these methods from the present $\mu$ s domain could shed much light on the intricate details of laser ionization.

There is some further evidence related indirectly to the laser target interaction. Crater depths $(78,202)$ and the depth of melting $(231)$ were measured and provide upper estimates of the overall material influx. Kinetic energy measurements of neutrals $(45,203,205,232)$ and of ions $(58-67,217-219,233)$ are still among the most effective methods in revealing information about their conditions of formation. As a rule, large kinetic energies observed at elevated irradiances indicate hydrodynamic acceleration, Coulomb explosion, or other high encrgy processes, whereas near thermal energy distributions are generated in low irradiance desorption experiments. Mass spectrometric peak shapes, even after second-order energy focusing, can reveal information about the initial kinetic energy and departing angle distributions of the ions $(225,241)$.

Ion yields and especially relative sensitivity factors are extensively covered in the literature $(78,202,234-236)$. Their interpretation requires extreme caution because, owing to ion discrimination effects, instrumental factors may distort the results $(53,237)$. One important feature of laser ionization is the remarkable uniformity of the relative sensitivity factors in a given sample at high irradiances (235). Furthermore, direct analysis of nonconducting materials is possible, since the excitation energy is coupled to the target through optical absorption. Methods to realize low molecular and cluster interference levels are under investigation (234).

Mathematical models of the redistribution and expansion phase have to include intense mass transport. Therefore, all three conservation equations should be treated simultaneously. Experience in plasma hydrodynamics is stemming from nuclear fusion and heavy ion source research (238-240). The calculations devoted to LIMS are based on the one-component, one-dimensional (1C-1D) model 

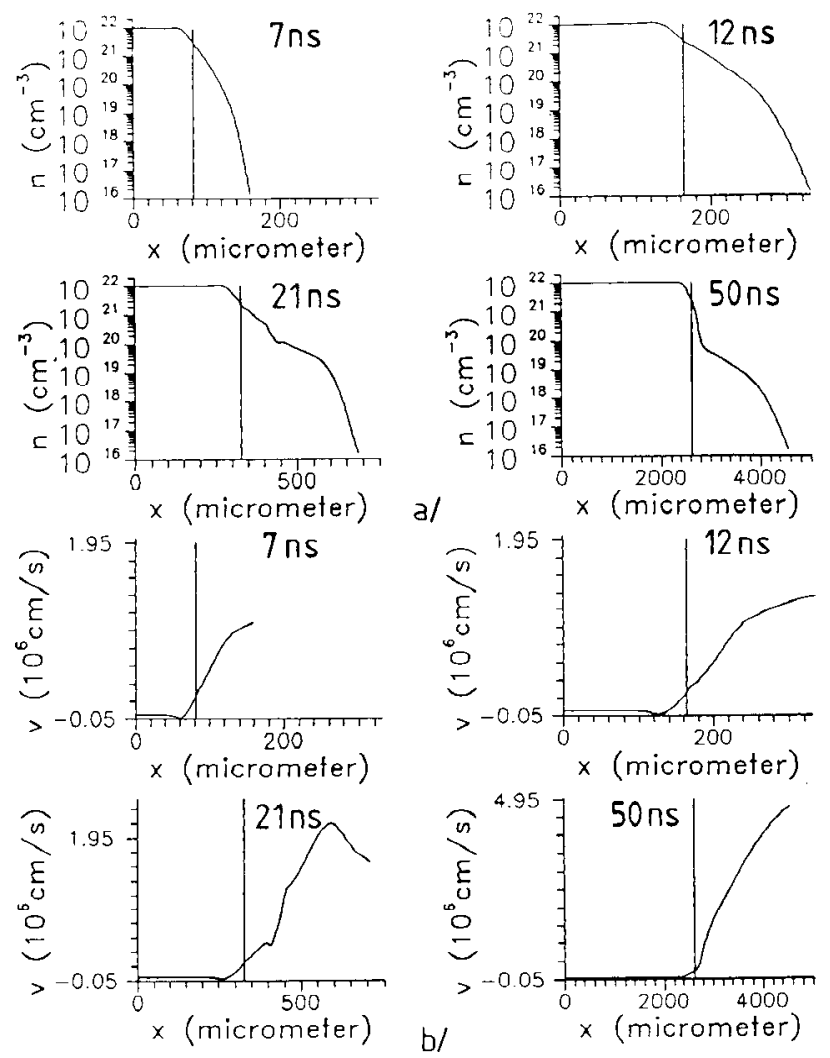

Figure 14. Time development of changes during a $50 \mathrm{~ns}$ high irradiance $\left(10^{9}\right.$ $W / \mathrm{cm}^{2}$ ) ruby laser pulse. The vertical line shows the surface of the target. (a) $\mathrm{lg}$ (density) and (b) velocity profiles at $7 \mathrm{~ns}, 12 \mathrm{~ns}, 21 \mathrm{~ns}$, and $50 \mathrm{~ns}$, respectively (72).

$(72,167,224)$. The model incorporates normal and plasma absorption mechanisms and local thermal equilibrium condition for ionization. Enhancement of ion formation in the case of a $10^{9} \mathrm{~W} / \mathrm{cm}^{2}$ ruby laser pulse impinging on carbon target is demonstrated in Figures 14 and 15. Dramatic differences between the 12 and 21 ns profile are attributed to the onset of self-amplifying plasma absorption mechanism.

A comprehensive study of the model covers three different laser types $\left(\mathrm{CO}_{2}\right.$, ruby and frequency-quadrupled Nd-YAG) and three classes of solid targets (metals, transparent, and opaque insulators). Successful comparison of gas cloud extensions with expansion velocity measurements and demonstrated threshold behavior of ion yields as a function of laser intensity provide support for the calculations. Strong contribution of the hydrodynamic acceleration to high energy ion formation is also confirmed.

Future trends within sight are easy to predict on the basis of accumulating experience and diagnostic results. It became clear that the requirements of both material ablation and ionization cannot be completely fulfilled with the same laser. 

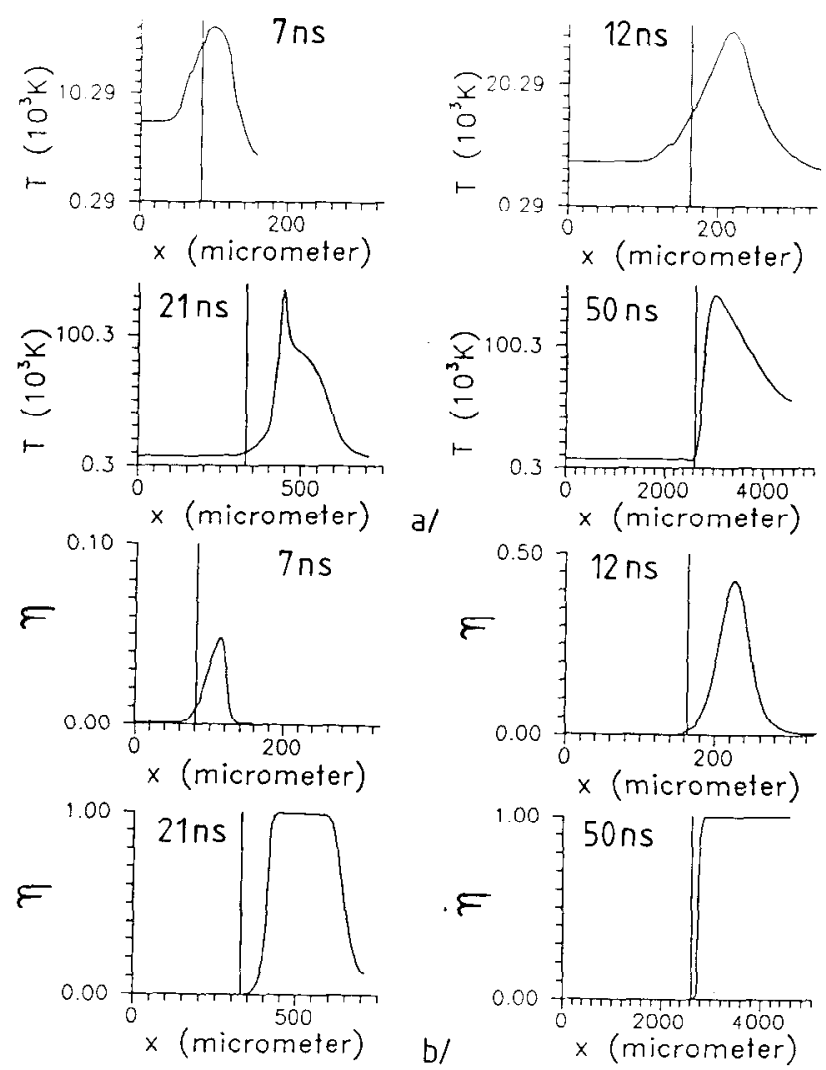

Figure 15. (a) temperature and (b) ionization degree profiles in the same calculation as in Fig. 13. Total ionization and resonant absorption is demonstrated on the curves corresponding to $21 \mathrm{~ns}$ and $50 \mathrm{~ns}$ (72).

More flexible and effective ion sources are expected from the introduction of two independent lasers. Thus, the wavelengths, irradiances, and the time difference of the two pulses can be adjusted to achieve better sensitivity. It is expected that ultraviolet or infrared lasers for ablation, shorter wavelength radiation for ionization, and matrix-assisted methods will become widely used.

\section{CONCLUDING REMARKS}

Promising developments of the different mass spectrometric techniques utilizing plasma generation for ionization in the last twenty years have led to their central place in the family of elemental analytical methods. Most of the plasmas involved have also been applied in optical emission spectroscopy, but the simplicity of the mass spectra provides competitive advantage in interpretation over the more complex optical spectra. Plasma diagnostics and modeling have been used for approximately the same amount of time to the benefit of ion source development. 
In elemental analysis, the plasmas fulfill a twofold requirement. They are capable of disintegrating the intra- and intermolecular bonds in the sample and at the same time are effective in ionizing the different species. New types of plasma ion sources are expected to emerge from those used in optical emission spectroscopy, in ion implantation, or from the techniques applied for ion generation in accelerators.

Combination methods will certainly have a large stake in future developments. Preliminary experiments already point to interest in the combination of lasers with inductively coupled plasma, lasers with glow discharge, lasers with ion beams, and lasers with other lasers (including RIMS) just to name the most commonplace possibilities.

As the role of diagnostics and modeling become more pronounced, the methods based on plasmas grow more sophisticated. Real-time examination of transient plasmas, although not yet routinely practiced, are within the reach of present measuring capabilities. More elaborate models can also be studied on large mainframe computers. Nonequilibrium treatment and two- or three-dimensional calculations are probably the best candidates for future expansion.

\section{REFERENCES}

1. Koppenaal, D. W. Anal. Chem. 1988, 60, 113R-131R.

2. Adams, F.; Gijbels, R.; Van Grieken, R., Eds. "Inorganic Mass Spectrometry"; Chem. Anal. Series No. 95; Wiley: New York, 1988.

3. Vályi, L. "Atom and Ion Sources"; Wiley \& Sons: London, 1977; pp. 127-230.

4. Tonegawa, A.; Shimoyama, T.; Yabe, E.; Takayama, K.; Takagi, K.; Fukui, R.; Kikuchi, R.; Okamoto, K.; Komiya, S. Nucl. Instr. Meth. 1987, B21, $212-214$.

5. Yabe, E. Rev. Sci. Instrum. 1987, 58, 1-5.

6. Yabe, E.; Tonegawa, A.; Satoh, D.; Takayama, K.; Fukui, R.; Takagi, K.; Okamoto, K.; Komiya, S. Vacuum 1986, 36, 43-45.

7. Yabe, E.; Fukui, R. Jpn. J. Appl. Phys. 1987, 26, 1179-1184.

8. Yabe, E.; Takeshiro, S.; Sunako, K.; Takayama, K.; Fukui, R.; Takagi, K.; Okamoto, K.; Komiya, S. Nucl. Instr. Meth. 1985, B6, 119-122.

9. Lejeune, C.; Grandchamp, J. P.; Kessi, O.; Gilles, J. P. Vacuum 1986, 36, 837-840.

10. Root, J.; Asmussen, J. Rev. Sci. Instrum. 1985, 56, 1511-1519.

11. Asmussen, J.; Dahimene, M. J. Vac. Sci. Technol. 1987, B5, 328-331.

12. Dahimene, M.; Asmussen, J. J. Vac. Sci. Technol. 1986, B4, 126-130.

13. Leung, K. N.; Kunkel, W. B. Phys. Rev. Lett. 1987, 59, 787-790.

14. Walther, S. R.; Leung, K. N.; Kunkel, W. B. Appl. Phys. Lett. 1987, 51, 566-568.

15. Nightingale, M. P. S.; Holmes, A. J. T.; Forrest, M. J.; Burgess, D. D. J. Phys. D: Appl. Phys. 1986, 19, 1707-1722.

16. Hopkins, M. B.; Graham, W. G. Vacuum 1986, 36, 873-876.

17. Piosczyk, B.; Dammertz, G. Rev. Sci. Instrum. 1986, 57, 840-846.

18. Hershcovitch, A. I.; Kovarik, V. J.; Prelec, K. Rev. Sci. Instrum. 1986, 57, 827-830.

19. Walther, S. R.; Leung, K. N. Vacuum 1986, 36, 869-871.

20. Alton, G. D.; Beckers, R. M.; Johnson, J. W. Nucl. Instr. Meth. 1986, A244, 148154.

21. Dreike, P. L.; Tisone, G. C. J. Appl. Phys. 1986, 59, 371-377.

22. Ishikawa, J.; Tsuji, H.; Takagi, T. Vacuum 1986, 36, 877-890.

23. Humphries, Jr. S.; Len, L. K. Rev. Sci. Instrum. 1986, 57, 2929-2934.

24. Koshelev, K. N.; Sidelnikov, Yu. V. Nucl. Instr. Meth. 1985, B9, 704-705.

25. Venogupalan, M., Ed. "Reactions Under Plasma Conditions" Vol. I; Wiley: New York, 1971, pp. 367-542. 
26. Drawin, H. W. In "Reactions Under Plasma Conditions" Vol. I; Venogupalan, M., Ed., Wiley: New York, 1971; pp. 53-238.

27. Sheeline, A. Spectrochim. Acta 1988, 43B, 57-62.

28. Emeleus, K. G.; Coulter, J. R. M. Int. J. Mass Spectrom. Ion Process. 1988, 83, 209-214.

29. Ref. 25. pp. 534-537.

30. Pei-q̨i, L.; Pei-zhong, G.; Tie-zheng, L.; Houk, R. S. Spectrochim. Acta 1988, 43B, 273 285.

31. Smy, P. R. Adv. Phys. 1976, 25, 517-553.

32. Ref. 25 pp. $389-446$.

33. Montaser, A.; Fassel, V. A.; Larsen, G. Appl. Spectrosc. 1981, 35, 385-389.

34. Furuta, N.; Horlick, G. Spectrochim. Acta 1982, 37, 53-64.

35. Montaser, A.; Van Hoven, R. L. CRC Crit. Rev. Anal. Chem. 1987, 18, 45-103.

36. Bonnie, J. H. M.; Eenshuistra, P. J.; Hopman, H. J. AIP Conference Proceedings No. 158., AIP: New York, 1987, pp. 133-142.

37. Chen, G.; Yeung, E. S. Anal. Chem. 1988, 60, 864-868.

38. Mermet, J. M. In "Inductively Coupled Plasma Emission Spectroscopy" Part 2; Boumans, P. W. J. M., Ed., Wiley: New York, 1988, pp. 353-386.

39. Savickas, P. J.; Hess, K. R.; Marcus, R. K.; Harrison, W. W. Anal. Chem. 1984, 56, 817819.

40. Harrison, W. W. In "Inorganic Mass Spectrometry"; Adams, F., Gijbels, R., Van Grieken, R., Eds., Chem. Anal. Series No. 95; Wiley: New York, 1988, pp. 85-123.

41. Turk, G. C.; Devoe, J. R.; Travis, J. C. Anal. Chem. 1982, 54, 643-645.

42. Travis, J. C.; Turk, G. C.; Green, R. B. Anal. Chem. 1982, 54, 1006A 1018A.

43. Kagawa, K.; Matsuda, Y.; Yokoi, S.; Nakajima, S. J. Anal. Atom. Spectr. 1988, 3, 415-419.

44. Gauthier, T. D.; Clarke, R. H.; Isner, J. M. J. Appl. Phys. 1988, 64, 2736-2741.

45. Spengler, B.; Bahr, U.; Karas, M.; Hillenkamp, F. Analytical Instrumentation 1988, 17, 173 193.

46. Houk, R. S.; Fassel, V. A.; Flesch, G. D.; Svec, H. J.; Gray, A. L.; Taylor, C. E. Anal. Chem. 1980, 52, 2283-2289.

47. Gray, A. L.; Date, A. R. Analyst 1983, 108, 1033-1050.

48. Douglas, D. J.; French, J. B. Spectrochim. Acta 1986, 41B, 197-204.

49. Houk, R. S.; Svec, H. J., Fassel, V. A. Appl. Spectrosc. 1981, 35, 380-384.

50. Gray, A. L. Spectrochim. Acta 1986, 41B, 151-167.

51. Olivares, J. A.; Houk, R. S. Anal. Chem. 1986, 58, 20-25.

52. Satzger, R. D.; Fricke, F. L.; Brown, P. G.; Caruso, J. A. Spectrochim. Acta 1987, 42B, 705-712.

53. De Wolf, M.; Mauney, T.; Michiels, E.; Gijbels, R. Scann. Electr. Microsc. 1986, III, 799 814.

54. Olivares, J. A.; Houk, R. S. Appl. Spectrosc. 1985, 39, 1070-1077.

55. Fulford, J. E.; Douglas, D. J. Appl. Spectrosc. 1986, 40, 971-974.

56. Woolston, J. R.; Honig, R. E. Rev. Sci. Instrum. 1964, 35, 69.

57. Ramendik, G. I.; Derzhiev, V. I.; Surkov, Yu. A.; Ivanova, V. F.; Grechishnikov, A. V.; Sysoev, A. A.; Oleinikov, V. A.; Aleksandrov, V. A. Int. J. Mass Spectrom. Ion Phys. 1981, 37, 331-339.

58. Demtröder, W.; Jantz, W. Plasma Physics 1970, 12, 691-703.

59. Dinger, R.; Rohr, K.; Weber, H. J. Phys. D: Appl. Phys. 1980, 13, 2301-2307.

60. Chowdhury, S. S.; Clement, R. M.; Miles, II. T. J. Phys. L: Sci. Instr. 1980, 13, 1099 1105.

61. Tabet, J.; Cotter, R. J. Int. J. Mass Spectrom. Ion Proc. 1983, 54, 151-158.

62. Van Der Peyl, G. J. Q.; Van Der Zande, W. J.; Bederski, K.; Boerboom, A. J. H.; Kistemaker, P. G. Int. J. Mass Spectrom. Ion Phys. 1983, 47, 7-10.

63. Van Der Peyl, G. J. Q.; Van Der Zande, W. J.; Kistemaker, P. G. Int. J. Mass Spectrom. Ion Proc. 1984, 62, 51-71.

64. Mauney, T.; Adams, F. Int. J. Mass Spectrom. Ion Proc. 1984, 59, 103-119.

65. Michiels, E.; Mauney, T.; Adams, F.; Gijbels, R. Int. J. Mass Spectrom. Ion Proc. 1984, 61, 231-246.

66. Tsong, T. T. Int. J. Mass Spectrom. Ion Proc. 1986, 70, 1-21. 
67. Vertes, A.; Juhasz, P.; Jani, P.; Czitrovszky, A. Int. J. Mass Spectrom. Ion Proc. 1988, 83, 45-70.

68. Viczian, M.; Cornides, I.; Van Puymbroeck, J.; Gijbels, R. Int. J. Mass Spectrom. Ion Phys. $1983,51,77-92$.

69. Van Puymbroeck, J.; Gijbels, R.; Viczian, M.; Cornides, I. Inl. J. Mass Spectrom. Ion Proc. $1984,56,269-280$.

70. Cotter, R. J.; Snow, M.; Colvin, M. In "Ion Formation from Organic Solids"; Benninghoven, A., Ed. Springer: Berlin, 1983, pp. 206-210.

71. Boulos, M. I.; Barnes, R. M. in "Inductively Coupled Plasma Emission Spectroscopy"; Boumans, P. W. J. M., Ed. Part 2; Wiley: New York, 1988, pp. 289-352.

72. Vertes, A.; Juhasz, P.; De Wolf, M.; Gijbels, R. Scanning Electron Microsc. 1988, 2, 1853 1877.

73. Holt, M. "Numerical Methods in Fluid Dynamics"; Springer: Berlin, 1977.

74. Verlinden, J.; Swenters, K.; Gijbels, R. Spectrochim. Acta 1984, 39B, 1573-1575.

75. van Straaten, M.; Melchers, F. G.; Swenters, K.; Beske, H. E.; Gijbels, R. Int. J. Mass Spectrom. Ion Proc. in press.

76. Swenters, K.; Verlinden, J.; Bernard, P.; Gijbels, R. Int. J. Mass Spectrom. Ion Proc. 1986, $71,85-102$.

77. Verlinden, J. A. A.; Swenters, K. M. E.; Gijbels, R. H. H. Anal. Chem. 1985, 57, 131136.

78. Kovalev, I. D.; Maksimov, G. A.; Suchkov, A. I.; Larin, N. V. Int. J. Mass Spectrom. Ion Phys. 1978, 27, 101-137.

79. Kohler, V. L.; Harris, A.; Wallach, E. R. "Third International Laser Microprobe Mass Spectrometry Workshop"; U.I.A.: Antwerp (Belgium), 1986; pp. 127-131.

80. Bruynseels, F.; Van Grieken, R. Int. J. Mass Spectrom. Ion Proc. 1986, 74, 161-177.

81. Musselman, I. H.; Linton, R. W.; Simons, D. S. Anal. Chem. 1988, 60, 110-114.

82. Cornides, I.; Gál, T. High Temp. Sci. 1978, 10, 171.

83. Gray, A. L. Analyst 1985, 110, 551-556.

84. Arrowsmith, P. Anal. Chem. 1987, 59, 1437-1444.

85. Edmonds, T. E.; Horlick, G. Appl. Spectrosc. 1977, 31, 536-541.

86. Bydder, F. L.; Miller, G. P. Spectrochim. Acta 1988, 43B, 819-829.

87. Van Der Muller, J. A. M.; Nowak, S.; Van Lemmeren, A. C. A. P.; Schram, D. C.; Van Der Sijde, B. Spectrochim. Acta 1988, 43B, 317-324.

88. Winge, R. K.; Eckels, D. E.; DeKalb, E. L.; Fassel, V. A. J. Anal. Atomic Spectr. 1988, 3, 849-855.

89. Miller, R. C.; Ayen, R. J. J. Appl. Phys. 1969, 40, 5260-5273.

90. Barnes, R. M.; Schleicher, R. G. Spectrochim. Acta 1975, 30B, 109-134.

91. Barnes, R. M.; Nikdel, S. J. Appl. Phys. 1976, 47, 3929-3934.

92. Barnes, R. M.; Schleicher, R. G. Spectrochim. Acta 1981, 36B, 81-101.

93. Mostaghimi, J.; Proulx, P.; Boulos, M. I.; Barnes, R. M. Spectrochim. Acta 1985, 40B, 153166.

94. Koppenaal, D. W.; Quinton, L. F. J. Anal. Atomic Spectr. 1988, 3, 667-672.

95. Douglas, D. J.; French, J. B. Anal. Chem. 1981, 53, 37-41.

96. Pertel, R. Int. J. Mass Spectrom. Ion Phys. 1975, 16, 39-52.

97. Olivares, J. A.; Houk, R. S. Anal. Chem. 1985, 57, 2674-2679.

98. Gray, A. L.; Houk, R. S.; Williams, J. G. J. Anal. Atomic Spectr. 1987, 2, 13-20.

99. Houk, R. S.; Schoer, J. K.; Crain, J. S. J. Anal. Atomic Spectr. 1987, 2, 283-286.

100. Horlick, G.; Tan, S. H.; Vaughan, M. A.; Rose, C. A. Spectrochim. Acta 1985, 40B, 15551572 .

101. Gray, A. L. Spectrochim. Acta 1985, 40B, 1525-1537.

102. Kawaguchi, H.; Tanaka, T.; Mizuike, A. Spectrochim. Acta 1988, 43B, 955-962.

103. Gray, A. L. J. Anal. Atomic Spectr. 1986, 1, 247-249.

104. Scott, R. H.; Fassel, V. A.; Kniseley, R. N.; Nixon, D. E. Anal. Chem. 1974, 46, 75-80.

105. Webb, B. D.; Denton, M. B. J. Anal. Atomic Spectr. 1987, 2, 21-26.

106. Houk, R. S.; Montaser, A.; Fassel, V. A. Appl. Spectrosc. 1983, 37, 425-428.

107. Montaser, A.; Chan, S.; Koppenaal, D. W. Anal. Chem. 1987, 59, 1240-1242.

108. Hasegawa, T.; Winefordner, D. J. Spectrochim. Acta 1987, 42B, 637-649. 
109. Faires, L. M.; Palmer, B. A.; Engleman, Jr. R.; Niemczyk, T. M. Spectrochim. Acta 1984, $39 B, 819-828$.

110. Zeeman, P. B.; Terblanche, S. P.; Visser, K.; Hamm, F. H. Appl. Spectrosc. 1978, 32, 572576.

111. Raeymaekers, B.; Broekaert, J. A. C.; Leis, F. Spectrochim. Acta 1988, 43B, 941-949.

112. Wilson, D. A.; Vickers, G. H.; Hieftje, G. M. Appl. Spectrosc. 1987, 41, 875-880.

113. Douglas, D. J.; French, J. B. J. Anal. Atomic Spectr. 1988, 3, 743-747.

114. Douglas, D. J.; Kerr, L. A. J. Anal. Atomic Spectr. 1988, 3, 749-752.

115. Gillson, G. R.; Douglas, D. J.; Fulford, J. E.; Halligan, K. W.; Tanner, S. D. Anal. Chem. 1988, 60, 1472.-1474.

116. Zhu, G.; Browner, R. F. J. Anal. Atomic Spectr. 1988, 3, 781-789.

117. Williams, J. G.; Gray, A. L.; Norman, P.; Ebdon, L. J. Anal. Atomic Spectr. 1987, 2, 469 472.

118. Raeymaekers, B.; Graule, T.; Broekaert, J. A. C.; Adams, F.; Tschöpel, P. Spectrochim. Acta 1988, 43B, 923-940.

119. Kawaguchi, H.; Asada, K.; Mizuike, A. Mikrochimica Acta 1988, III, 143-152.

120. Lim, H. B.; Houk, R. S.; Edelson, M. C.; Carney, K. P. J. Anal. Atomic Spectr. 1989, in press.

121. Lim, H. B.; Houk, R. S.; Crain, J. S. Spectrochim. Acta B 1989, in press.

122. Beenakker, C. I. M. Spectrochim. Acta 1976, 31B, 483-486.

123. Haas, D. L.; Carnahan, J. W.; Caruso, J. A. Appl. Spectrosc. 1983, 37, 82-85.

124. Skogerboe, R. K.; Coleman, G. N. Anal. Chem. 1976, 48, 611A-622A.

125. Zander, A. T.; Hieftje, G. M. Appl. Spectrosc. 1981, 35, 357-371.

126. Bollo-Kamara, A.; Codding, E. G. Spectrochim. Acta 1981, 36B, 973-982.

127. Haas, D. L.; Caruso, J. A. Anal. Chem. 1984, 56, 2014-2019.

128. Tanabe, K.; Haraguchi, H.; Fuwa, K. Spectrochim. Acta 1983, 38B, 49-60.

129. Brown, P. G.; Brotherton, T. J.; Workman, J. M.; Caruso, J. A. Appl. Spectrosc. 1987, 41, 774-779.

130. Workman, J. M.; Fleitz, P. A.; Fannin, H. B.; Caruso, J. A.; Seliskar, C. J. Appl. Spectrosc. 1988, 42, 96-100.

131. Besner, A.; Moisan, M.; Hubert, J. J. Anal. Atomic Spectr. 1988, 3, 863-866.

132. Douglas, D. J.; Quan, F. S. K.; Smith, R. G. Spectrochim. Acta 1983, 38B, 39-48.

133. Satzger, R. D.; Fricke, F. L.; Caruso, J. A. J. Anal. Atomic Spectr. 1988, 3, 319-323.

134. Brown, P. G.; Davidson, T. M.; Caruso, J. A. J. Ancl. Atomic Spectr. 1988, 3, 763769 .

135. Creed, J. T.; Mohamad, A. H.; Davidson, T. M.; Ataman, G.; Caruso, J. A. J. Anal. Atomic Spectr. 1988, 3, 923-926.

136. Poussel, E.; Mermet, J. M.; Deruaz, D.; Beaugrand, C. Anal. Chem. 1988, 60, 923-927.

137. Chapman, B. "Glow Discharge Processes"; Wiley: New York, 1980.

138. Harrison, H. W.; Bentz, B. L. Prog. Anal. Spectrosc. 1988, 11, 53-110.

139. Harrison, W. W.; Magee, C. W. Anal. Chem. 1974, 46, 461-464.

140. Colby, B. N.; Evans, Jr. C. A. Anal. Chem. 1974, 46, 1236-1242.

141. Coburn, J. W.; Eckstein, E. W.; Kay, E. J. Appl. Phys. 1975, 46, 2828-2830.

142. Mattson, W. A.; Bentz, B. L.; Harrison, W. W. Anal. Chem. 1976, 48, 489-491.

143. Harrison, W. W.; Bentz, B. L. Anal. Chem. 1979, 51, 1853-1855.

144. Loving, T. J.; Harrison, W. W. Anal. Chem. 1983, 55, 1526-1530.

145. Foss, G. O.; Svec, H. J.; Conzemius, R. J. Anal. Chim. Acta 1983, 147, 151-162.

146. Marcus, R. K.; King, Jr. F. L.; Harrison W. W. Anal. Chem. 1986, 58, 972-974.

147. Jakubowski, N.; Stuewer, D.; Toelg, G. Int. I. Mass Spectrom. Ion Proc. 1986, 71, 183-197.

148. Harrison, W. W. J. Anal. Atomic Spectr. 1988, 3, 867-872.

149. Davis, W. D.; Vanderslice, T. A. Phys. Rev. 1963, 131, 219-228.

150. Winters, H. F. In "Plasma Chemistry III"; Vepřek, S.; Venugopalan, M., Eds., Springer: Berlin, 1980, pp. 69-125.

151. Abril, I.; Gras-Marti, A.; Vallés-Abarca, J. A. Phys. Rev. A 1983, 28, 3677-3678.

152. Abril, I.; Gras-Marti, A.; Vallés-Abarca, J. A. Phys. Rev. D 1984, 17, 1841-1849.

153. Abril, I. Comp. Phys. Comm. 1988, 51, 413-422.

154. Ben-Amar, A.; Erez, G.; Fastig, S.; Shuker, R. Appl. Optics 1984, 23, 4529-4531. 
155. Harrison, W. W.; Hess, K. R.; Marcus, R. K.; King, F. L. Anal. Chem. 1986, 58, 341A$356 \Lambda$.

156. Ref. 2. pp. 85-123.

157. Loving, T. J.; Harrison, W. W. Anal. Chem. 1983, 55, 1523-1526.

158. Hess, K. R.; Harrison, W. W. Anal. Chem. 1988, 60, 691-696.

159. Bruhn, C. G.; Bentz, B. L.; Harrison, W. W. Anal. Chem. 1978, 50, 373-375.

160. Bruhn, C. G.; Bentz, B. L.; Harrison, W. W. Anal. Chem. 1979, 51, 673-678.

161. Cantle, J. E.; Hall, E. F.; Shaw, C. J.; Turner, P. J. Int. J. Mass Spectrom. Ion Phys. 1983, $46,11-13$

162. Jakobowski, N.; Stuewer, D.; Vieth, W. Anal. Chem. 1987, 59, 1825-1830.

163. King, F. L.; McCormack, A. L.; Harrison, W. W. J. Anal. Atomic Spectr. 1988, 3, 883-886.

164. Valles-Abarca, J. A.; Gras-Marti, A. J. Appl. Phys. 1984, 55, 1370-1378.

165. Ashcroft, N. W.; Mermin, N. D. "Solid State Physics"; Holt, Reinhart and Winston: New York, 1976, pp. 319-320.

166. Majidi, V.; Hsu, W.; Coleman, D. M. Spectrochim. Acta 1988, 43B, 561-573.

167. Vertes, A.; De Wolf, M.; Juhasz, P.; Gijbels, R. Anal. Chem. 1989, 61, 1029-1035.

168. Früngel, F. B. A. "High Speed Pulse Technology" Vol. IV; "Sparks and I aser Pulses"; Academic: New York, 1980.

169. Ref. 168 pp. 276-279.

170. Bacon, J. R.; Ure, A. M. Analyst 1984, 109, 1229-1254.

171. Ref. 2 pp. 17-84.

172. Ramendik, G. I. Adv. Mass Spectrom. 1980, 8A, 408-413.

173. Ramendik, G. I.; Kryuchkova, O. I.; Tyurin, D. A.; Mchedlidze, T. R.; Kaviladze, M. Sh. Int. J. Mass Spectrom. Ion Proc. 1985, 63, 1-15.

174. Franzen, J. in Ahearn, A. J., Ed., "Trace Analysis by Mass Spectrometry"; Academic: New York, 1972, p. 11.

175. Maitland, A. J. Appl. Phys. 1961, 32, 2399-2407.

176. Davies, D. K.; Biondi, M. A. J. Appl. Phys. 1966, 37, 2969-2977.

177. Kelsey, T. J. Phys. D: Appl. Phys. 1972, 5, 569-574.

178. Suzuki, K.; Kobayashi, S. J. Phys. D: Appl. Phys. 1982, 15, 1227-1230.

179. Davies, D. K.; Biondi, M. A. J. Appl. Phys. 1970, 41, 88-93.

180. Davies, D. K.; Biondi, M. A. J. Appl. Phys. 1971, 42, 3089-3107.

181. Derzhiev, V. I.; Ramendik, G. I.; Liebich, V.; Mai, H. Int. J. Mass Spectrom. Ion Phys. 1980, 32, 345-361.

182. Chalmers, I. D.; Phukan, B. D. Vacuum 1982, 32, 145-150.

183. Verlinden, J.; Van Puymbroeck, J.; Gijbels, R. Int. J. Mass Spectrom. Ion Phys. 1983, 47, 287-290.

184. Van Puymbroeck, J.; Verlinden, J.; Swenters, K.; Gijbels, R. Talanta 1984, 31, 177184.

185. Stauffer, D. "Introduction to Percolation Theory"; Taylor \& Francis: London, 1985.

186. Swenters, K. M.; Verlinden, J. A.; Gijbels, R. H. Adv. Mass Spectrom. 1986, 10B, 10071008 .

187. Haemers, J. Spectrochim. Acta 1983, 38B, 1367-1370.

188. Haemers, I. Spectrochim. Acta 1983, 38B, 859-863.

189. Helmer, D. J. C.; Walters, J. P. Appl. Spectrosc. 1984, 38, 399-405.

190. Liu, X. D.; Raeymaekers, B.; Van Espen, P.; Adams, F. Anal Chim. Acta 1987, 195, 181192.

191. Raeymakers, B.; Van Espen, P.; Adams, F.; Broekaert, J. A. C. Appl. Spectrosc. 1988, 42, 142-150.

192. Datta, B. P.; Jain, H. C. Int. J. Mass Spectrom. Ion Proc. 1986, 68, 219-237.

193. Galburt, V. A.; Zelenin, A. E.; Sikharulidze, G. G. Int. J. Mass Spectrom. Ion Proc. 1983/1984, $55,125-132$.

194. Demortier, G.; Lamberts, L. Int. J. Mass Spectrom. Ion. Phys. 1979, 31, 135-150.

195. Berthod, J.; Andreani, A.; Stefani, R. Int. J. Mass Spectrom. Ion Phys. 1978, 27, 305-317.

196. Gerasimov, V. A.; Chupakhin, M. S.; Shelpakova, I. R. I. Anal. Chem. USSR 1980, 35, 138, translated from $\mathrm{Zh}$. Anal. Khim. 1980, 35, 224.

197. Wieth, W. Spectrochim. Acta 1985, 42B, 1085-1092. 
198. Ramendik, G. I.; Manzon, B. M.; Tyurin, D. A.; Benyaev, N. E.; Komleva, A. A. Talanta 1987, 34, 61-67.

199. Adams, F. Phil. Trans. R. Soc. Lond. 1982, A 305, 509-519.

200. Pustovit, A. N.; Sikharulidze, G. G. Mikrochimica Acta 1981, II, 219-227.

201. Shibanov, A. N. In "Laser Analytical Spectrochemistry"; Letokov, V. S., Ed. Adam Hilger: Bristol and Boston, 1986, pp. 353-404.

202. Conzemius, R. J.; Capellen, J. M. Int. J. Mass Spectrom. Ion Phys. 1980, 34, 197-271.

203. Estler, R. C.; Apel, E. C.; Nogar, N. S. J. Opt. Soc. Am. B 1987, 4, 281-286.

204. Antonov, V. S.; Lethokov, V. S.; Shibanov, A. N. Appl. Phys. 1981, 25, 71-76.

205. Namiki, A.; Fukano, H.; Kawai, T.; Yasuda, Y.; Nakamura, T. J. Phys. Soc. Jpn. 1985, $54,3162-3167$.

206. Karas, M.; Bachmann, D.; Hillenkamp, F. Anal. Chem. 1985, 57, 2935-2939.

207. Karas, M.; Bachmann, D.; Bahr, U.; Hillenkamp, F. Int. J. Mass Spectrom. Ion Proc. 1987, $78,53-68$.

208. Ichige, K.; Matsumoto, Y.; Namiki, A. Nucl. Instr. Meth. 1988, B33, 820-823.

209. Lax, M. I. Appl. Phys. 1977, 48, 3919-3924.

210. Bartholomeusz, B. J. J. Appl. Phys. 1988, 64, 3815--3819.

211. Lax, M. Appl. Phys. Lett. 1978, 33, 786-788.

212. Lucchese, R. R.; Tully, J. C. J. Chem. Phys. 1984, 81, 6313-6319.

213. Lim, C.; Tully, J. C. J. Chem. Phys. 1986, 85, 7423-7433.

214. Chiarelli, M. P.; Gross, M. L. Int. J. Mass Spectrum. Ion Proc. 1987, 78, 37-52.

215. Hercules, D. M.; Balasanmugam, K.; Dang, T. A.; Li, C. P. Anal. Chem. 1982, 54, 280A$305 \mathrm{~A}$.

216. von Allmen, M. "Laser-Beam Interactions with Materials"; Springer Ser. in Materials Sci. 2,; Springer: Berlin, 1987, pp. 146-200.

217. Tallens, G. J. Plasma Phys. 1980, 22, 709-718.

218. Tallens, G. J. Optics Comm. 1981, 37, 108-112.

219. Goto, T.; Kishi, K.; Okuda, T. Kaku Yugo Kenkyu 1982, 47, 592-599.

220. Viswanadham, S. K.; Hercules, D. M.; Schreiber, E. M.; Weller, R. R.; Giam, C. S. Anal. Chem. 1988, 60, 2346-2353.

221. Fürstenau, N.; Hillenkamp, F.; Nitsche, R. Int. J. Mass Spectrom. Ion Phys. 1979, 31, 8591.

222. Fürstenau, N.; I lillenkamp, F. Int. J. Mass Spectrom. Ion Phys. 1981, 37, 135-151.

223. Becker, S.; Dietze, H. J. ZFI-Mitteilungen 1987, 134, 25-48.

224. Vertes, A.; Juhasz, P.; De Wolf, M.; Gijbels, R. Int. J. Mass Spectrom. Ion Proc. 1989, in press.

225. Vertes, A.; Juhasz, P.; Matus, L. Int. J. Mass Spectrom. Ion Proc. 1986, 73, 109-125.

226. Pitsch, P.; Rohr, K.; Weber, H. J. Phys. D: Appl. Phys. 1981, 41, L51-L55.

227. Muroo, K.; Nakano, N.; Kuroda, H. Laser Interact. Relat. Phenom. 1986, 7, 791 802.

228. Huie, C. W.; Yeung, E. S. Spectrochim. Acta 1985, 40B, 1255-1258.

229. Yappert, M. C.; Kimbrell, S. M.; Yeung, E. S. Appl. Optics 1987, 26, 3536-3541.

230. Kimbrell, S. M.; Yeung, E. S. Spectrochim. Acta 1988, 43B, 529-534.

231. Narayan, J. Appl. Phys. Lett. 1979, 34, 312-315.

232. Koppmann, R.; Refaei, S. M.; Pospieszcyk, A. J. Vac. Sci. Technol. 1986, A4, 79-85.

233. Dinger, R.; Rohr, K.; Weber, H. Laser and Particle Beams 1986, 4, $239-247$.

234. Jochum, K. P.; Matus; L.; Seufert, H. M. Fresenius Z. Anal. Chem. 1988, 331, 136-139.

235. Matus, L.; Seufert, M.; Jochum, K. P. Int. J. Mass Spectrom. Ion Proc. 1988, 84, 101-111.

236. Huang, L. Q.; Conzemius, R. J.; Houk, R. S. Appl. Spectrosc. 1987, 41, 667-670.

237. Michiels, E.; De Wolf, M.; Gijbels, R. Scan. Electr. Microsc. 1985, 1II, 947-958.

238. Dawson, J.; Kaw, P.; Green, B. Phys. Fluids 1969, 12, 875-882.

239. Mulser, P. Z. Naturforsch. 1970, 25A, 282-295.

240. Yasuda, H.; Sekiguchi, T. Jpn. J. Appl. Phys. 1979, 18, 2245-2254.

241. Vertes, A.; Juhasz, P.; Balazs, L.; De Wolf, M.; Gijbels, R. Int. J. Mass Spectrom. Ion Proc. $1988,84,255-269$. 